\title{
Bow shock nebulae of hot massive stars in a magnetized medium
}

\author{
D. M.-A. Meyer ${ }^{1} *$ A. Mignone,${ }^{2}$ R. Kuiper, ${ }^{1}$ A. Raga $^{3}$ and W. Kley ${ }^{1}$ \\ ${ }^{1}$ Institut für Astronomie und Astrophysik, Universität Tübingen, Auf der Morgenstelle 10, 72076 Tübingen, Germany \\ ${ }^{2}$ Dipartimento di Fisica Generale Facoltà di Scienze M.F.N., Università degli Studi di Torino, Via Pietro Giuria 1, 10125 Torino, Italy \\ ${ }^{3}$ Instituto de Ciencias Nucleares, Universidad Nacional Autónoma de México, Ap. 70-543, 04510 D.F., México
}

Received 2015; accepted 2015

\begin{abstract}
A significant fraction of OB-type, main-sequence massive stars are classified as runaway and move supersonically through the interstellar medium (ISM). Their strong stellar winds interact with their surroundings where the typical strength of the local ISM magnetic field is about 3.5-7 $\mu \mathrm{G}$, which can result in the formation of bow shock nebulae. We investigate the effects of such magnetic fields, aligned with the motion of the flow, on the formation and emission properties of these circumstellar structures. Our axisymmetric, magneto-hydrodynamical simulations with optically-thin radiative cooling, heating and anisotropic thermal conduction show that the presence of the background ISM magnetic field affects the projected optical emission our bow shocks at $\mathrm{H} \alpha$ and [OIII] $\lambda 5007$ which become fainter by about 1-2 orders of magnitude, respectively. Radiative transfer calculations against dust opacity indicate that the magnetic field slightly diminishes their projected infrared emission and that our bow shocks emit brightly at $60 \mu \mathrm{m}$. This may explain why the bow shocks generated by ionizing runaway massive stars are often difficult to identify. Finally, we discuss our results in the context of the bow shock of $\zeta$ Ophiuchi and we support the interpretation of its imperfect morphology as an evidence of the presence of an ISM magnetic field not aligned with the motion of its driving star.
\end{abstract}

Key words: methods: numerical - MHD - circumstellar matter - stars: massive.

\section{INTRODUCTION}

Massive star formation is a rare event that strongly impacts the whole Galactic machinery. These stars can release strong winds and ionizing radiation which shape their close surroundings into beautiful billows of swept-up and irradiated interstellar gas, that, in the case of a static or a slowly-moving star, can produce structures such as the Bubble Nebula (NGC 7635) in the constellation of Orion (Moore et al. 2002). The detailed study of the circumstellar medium of these massive stars provides us an insight into their internal physics (Langer 2012), it provides information on their intrinsic rotation (Langer et al. 1999), their envelope's (in)stability (Yoon \& Cantiello 2010) and allows us to understand the properties of their close surroundings throughout their evolution (van Marle et al. 2006; Chita et al. 2008) and after their death (Orlando et al. 2008; Chiotellis et al. 2012). This information is relevant for evaluating their feedback, i.e. the amount of energy, momentum and metals that massive stars inject into the interstellar medium (ISM) of the Galaxy (Vink 2006).

In particular, the bow shocks that develop around some fastmoving massive stars ejected from their parent stellar clusters pro-

* E-mail: dominique.meyer@uni-tuebingen.de vide an opportunity to constrain both their wind and local ISM properties (Huthoff \& Kaper 2002; Mever et al. 2014). Over the past decades, stellar wind bow shocks have first been serendipitously noticed as bright [OIII] $\lambda 5007$ spectral line arc-like shapes and/or distorted bubbles surrounding some massive stars having a particularly large space velocity with respect to their ambient medium. As a textbook example of such a bow shock, we refer the reader, e.g. to $\zeta$ Ophiuchi (Gull \& Sofia 1979, see Fig. 13 below). Further infrared observations, e.g. with the Infrared Astronomical Satellite (IRAS, Neugebauer et al. 1984) and the Wide-Field Infrared Satellite Explorer ( WISE, Wright et al.2010) facilities have made possible the compilation of catalogues of dozens of these bow shock nebulae (van Buren \& McCray 1988a; van Buren et al. 1995; Noriega-Crespo et al. 1997) and have motivated early numerical simulations devoted to the parsec-scale circumstellar medium of moving stars (Brighenti \& D'Ercole 1995a b). Recently, modern facilities led to the construction of multi-wavelengths databases, see e.g. the E-BOSS catalog (Peri et al. 2012, 2015) or the recent study of Kobulnicky et al. (2016). Moreover, a connection with high-energy astrophysics has been established, showing that stellar wind bow shocks produce cosmic rays in the same way as the expanding shock waves of growing supernova remnants do (del Valle et al. 2015). 
It is the discovery of bow shocks around the historical stars Betelgeuse (Noriega-Crespo et al. 1997) and Vela-X1 (Kaper et al. 1997) that revived the interest of the scientific community regarding such circumstellar structures generated by massive stars. The fundamental study of Comerón \& Kaper (1998) demonstrates that complex morphologies can arise from massive stars' wind-ISM interactions. Bow shocks are subject to a wide range of shear-like and non-linear instabilities (Blondin \& Koerwer 1998) producing severe distortions of their overall forms, which can only be analytically approximated (Wilkin 1996) in the particular situations of either a star moving in a relatively dense ISM Comerón \& Kaper 1998) or a high-mass star hypersonically moving through the Galactic plane (Meyer et al. 2014, hereafter Paper I). Tayloring numerical models to runaway red supergiant stars allows us to constrain the mass loss and local ISM properties of Betelgeuse (van Marle et al. 2011; Cox et al. 2012; Mackey et al. 2012) or IRC-10414 (Gvaramadze et al. 2014; Mever et al. 2014). For the sake of simplicity, these models neglect the magnetisation of the ISM.

However, magnetic fields are an essential component of the ISM of the Galaxy, e.g. its large scale component has a tendency to be aligned with the galactic spiral arms (Gaensler 1998). If the strength of the ISM magnetic field can reach up to several tenths of Gauss in the center of our Galaxy (see Rand \& Kulkarni 1989; Ohno \& Shibata 1993; Opher et al. 2009; Shabala et al. 2010), it can be even stronger in the cold phase of the ISM (Crutcher et al. 1999). In particular, radio polarization measures of the magnetic field in the context of Galactic ionized supershells are reported to be 2-6 $\mu \mathrm{G}$ in Harvey-Smith et al. (2011). This value is in accordance with previous estimates of the field strength in the warm phase of the ISM (Troland \& Heiles 1986) and was supported by hydrodynamical simulations (Fiedler \& Mouschovias 1993). Such a background magnetic field should therefore be included in realistic models of circumstellar nebulae around massive stars.

Numerical studies of magneto-hydrodynamical flows around an obstacle is approximated in the plane-parallel approach in de Sterck et al. (1998); de Sterck \& Poedts (1999). A significant number of circumstellar structures, such as the vicinity of our Sun (Pogorelov \& Semenov 1997), planetary nebulae developing in the vicinity of intermediate-mass stars (Heiligman 1980) or supernova remnants (Rozyczka \& Tenorio-Tagle 1995) have been studied in such a two-dimensional approach (see also Soker \& Dgani 1997; Pogorelov \& Matsuda 2000). The presence of a weak magnetic field can inhibit the growth rate of shear instabilities in the bow shocks around cool stars such as the runaway red supergiant Betelgeuse in the constellation of Orion (van Marle et al. 2014). We place our work in this context, focusing on bow shocks generated by hot, fast winds of mainsequence massive stars.

In this study, we continue our investigation of the circumstellar medium of runaway massive stars moving within the plane of the Milky way (Paper I, Meyer et al. 2015, 2016). As a logical extension of them, we present magneto-hydrodynamical models of a sample of some of the most common main-sequence, runaway massive stars (Kroupa 2001) moving at the most probable space velocities (Eldridge et al. 2011). We ignore any intrinsic inhomogenity or turbulence in the ISM. Particularly, we assume an axisymmetric magnetisation of the ISM surrounding the bow shocks in the spirit of van Marle et al. (2014). We concentrate our efforts on an initially $20 \mathrm{M}_{\odot}$ star, however, we also consider bow shocks generated by lower and higher initial mass stars. This project principally differs from Paper I because of (i) the inclusion of an ISM back- ground magnetic field leads to anisotropic heat conduction (see, e.g. Balsara et al. 2008) and (ii) our study does not concentrate on the secular stellar wind evolution of our bow-shock-producing stars. Note that our study introduces a reduced number of representative models due to the high numerical cost of the magnetohydrodynamical simulations. Following Acreman et al. (2016), we additionally appreciate the effects of the ISM magnetic field on the bow shocks with the help of radiative transfer calculations of dust continuum emission.

This paper is organised as follows. We start in Section 2 with a review of the physics included in our models for both the stellar wind and the ISM. We also recall the adopted numerical methods. Our models of bow shocks generated by main-sequence, runaway massive stars moving in a magnetised medium are presented together with a discussion of their morphology and internal structure in Section 3. We detail the emission properties of our bow shocks and discuss their observational implications in Section 4. Finally, we formulate our conclusions in Section 5.

\section{METHOD}

In the present section, we briefly summarise the numerical methods and microphysics utilised to produce magneto-hydrodynamical bow shock models of the circumstellar medium surrounding hot, runaway massive stars.

\subsection{Governing equations}

We consider a magnetised flow past a source of hot, ionized and magnetized stellar wind. The dynamics are described by the ideal equations of magneto-hydrodynamics and the dissipative character of the thermodynamics originates from the treatment of the gas with heating and losses by optically-thin radiation together with electronic heat conduction. These equations are,

$$
\begin{gathered}
\frac{\partial \rho}{\partial t}+\nabla \cdot(\rho \boldsymbol{v})=0, \\
\frac{\partial \boldsymbol{m}}{\partial t}+\boldsymbol{\nabla} \cdot\left(\boldsymbol{m} \otimes \boldsymbol{v}+\boldsymbol{B} \otimes \boldsymbol{B}+\hat{\boldsymbol{I}} p_{\mathrm{t}}\right)=\mathbf{0}, \\
\frac{\partial E}{\partial t}+\boldsymbol{\nabla} \cdot\left(\left(E+p_{\mathrm{t}}\right) \boldsymbol{v}-\boldsymbol{B}(\boldsymbol{v} \cdot \boldsymbol{B})\right)=\zeta(T, \rho, \mu),
\end{gathered}
$$

and,

$$
\frac{\partial \boldsymbol{B}}{\partial t}+\nabla \cdot(\boldsymbol{v} \otimes \boldsymbol{B}-\boldsymbol{B} \otimes \boldsymbol{v})=\mathbf{0},
$$

where $\rho$ and $\boldsymbol{v}$ are the mass density and the velocity of the plasma. In the relation of momentum conservation Eq. (2), the quantity $\boldsymbol{m}=\rho \boldsymbol{v}$ is the linear momentum of a gas element, $\boldsymbol{B}$ the magnetic field, $\hat{\boldsymbol{I}}$ the identity matrix and,

$$
p_{\mathrm{t}}=p+\frac{\boldsymbol{B} \cdot \boldsymbol{B}}{2}
$$

is the total pressure of the gas, i.e. the sum of its thermal component $p$ and its magnetic contribution $(\boldsymbol{B} \cdot \boldsymbol{B}) / 2$, respectively. Eq. (3) describes the conservation of the total energy of the gas,

$$
E=\frac{p}{(\gamma-1)}+\frac{\boldsymbol{m} \cdot \boldsymbol{m}}{2 \rho}+\frac{\boldsymbol{B} \cdot \boldsymbol{B}}{2}
$$

where $\gamma$ is the adiabatic index, which is taken to be $5 / 3$, i.e. we assume an ideal gas. The right-hand source term $\zeta(T, \rho, \mu)$ in Eq. 3. 
represents (i) the heating and the losses by optically-thin radiative processes and (ii) the heat transfers by anisotropic electronic thermal conduction (see Section 2.3). Finally, Eq. (4) is the induction equation and governs the time evolution of the vector magnetic field $\boldsymbol{B}$. The relation,

$$
c_{\mathrm{s}}=\sqrt{\frac{\gamma p}{\rho}},
$$

closes the system Eq. (1) - (4), where $c_{\mathrm{s}}$ denotes the adiabatic speed of sound.

\subsection{Boundary conditions and numerical scheme}

We solve the above described system of equations Eqs. (1) - (7) using the open-source PLUTO code1 (Mignone et al. 2007, 2012) on a uniform two-dimensional grid covering a rectangular computational domain in a cylindrical frame of reference $(O ; R, z)$ of origin $O$ and symmetry axis about $R=0$. The grid $\left[O ; R_{\max }\right] \times$ $\left[-z_{\min } ; z_{\max }\right]$ where $R_{\max },-z_{\min }$ and $z_{\max }$ are the upper and lower limits of the $O R$ and $O z$ directions, respectively, which are discretised with $N_{\mathrm{R}}=2 N_{\mathrm{z}}=1000$ cells such that the grid resolution is $\Delta_{R}=\Delta_{z}=R_{\max } / N_{\mathrm{R}}$. Learning from previous bow shock models (Comerón \& Kaper 1998; van Marle et al. 2006), we impose inflow boundary conditions corresponding to the stellar motion at $z=z_{\max }$ whereas outflow boundaries are set at $R=R_{\max }$ and $z=-z_{\min }$. Moreover, the stellar wind is modelled setting inflow boundaries conditions centered around the origin (see Section 2.4.

We integrate the system of partial differential equations within the eight-wave formulation of the magneto-hydrodynamical Eqs. (1)- 7), using a cell-centered representation consisting in evaluating $\rho, \boldsymbol{m}, E$ and $\boldsymbol{B}$ using the barycenter of the cells (see section 2 of Paper I). This formulation, used together with the Harten-Lax-van Leer approximate Riemann solver (Harten et al. 1983), conserves the divergence-free condition $\boldsymbol{\nabla} \cdot \boldsymbol{B}=\mathbf{0}$. The method is a second order, unsplit, time-marching algorithm scheme controlled by the Courant-Friedrich-Levy parameter initially set to $C_{\mathrm{cfl}}=0.1$. The gas cooling and heating rates are linearly interpolated from tabulated cooling curves (see Section 2.3 and the corresponding rate of change is subtracted from the total energy $E$. The parabolic term of heat conduction is integrated with the SuperTime-Stepping algorithm (Alexiades et al. 1996).

\subsection{Gas microphysics}

The source term $\zeta(T, \rho, \mu)$ in Eq. (3) represents the non-ideal thermodynamics processes that we take into account, and reads,

$$
\zeta(T, \rho, \mu)=\Phi(T, \rho)+\nabla \cdot \boldsymbol{F}_{\mathbf{c}}
$$

where $\Phi(T, \rho)$ is a function that stands for the processes by optically-thin radiation where,

$$
T=\mu \frac{m_{\mathrm{H}}}{k_{\mathrm{B}}} \frac{p}{\rho},
$$

is the gas temperature, with $\mu=0.61$ the mean molecular weight of the gas, $k_{\mathrm{B}}$ the Boltzmann constant and $m_{\mathrm{H}}$ the proton mass, respectively. The gain and losses by optically-thin radiative processes are taken into account via the following law,

$$
\Phi(T, \rho)=n_{\mathrm{H}} \Gamma(T)-n_{\mathrm{H}}^{2} \Lambda(T),
$$

${ }^{1}$ http://plutocode.ph.unito.it/ where $\Lambda(T)$ and $\Gamma(T)$ are the rate of change of the gas internal energy induced by heating and cooling as a function of $T$, respectively, and where $n_{\mathrm{H}}=\rho / \mu\left(1+\chi_{\mathrm{He}, \mathrm{Z}}\right) m_{\mathrm{H}}$ is the hydrogen number density with $\chi_{\mathrm{He}, \mathrm{Z}}$ the mass fraction of the coolants heavier than hydrogen. Details about the processes included into the cooling $\Lambda(T)$ and heating $\Gamma(T)$ laws are given in section 2 of Paper I.

The divergence term in the source function in Eq. 8) represents the anisotropic heat flux,

$$
\boldsymbol{F}_{\mathbf{c}}=\kappa_{\|} \hat{\boldsymbol{b}}(\hat{\boldsymbol{b}} \cdot \nabla T)+\kappa_{\perp}(\nabla T-\hat{\boldsymbol{b}} \cdot \nabla T),
$$

where $\hat{\boldsymbol{b}}=\boldsymbol{B} /\|\boldsymbol{B}\|$ is the magnetic field unit vector. It is calculated through the interface of the nearest neighbouring cells in the whole computational domain according to the temperature difference $\Delta T$ and to the local field orientation $\hat{\boldsymbol{b}}$ (see appendix of Mignone et al. 2012). The coefficients $\kappa_{\|}$and $\kappa_{\perp}$ are the heat coefficients along the directions parallel and normal to the local magnetic field streamline, respectively. Along the direction of the local magnetic field,

$$
\kappa_{\|}=K_{\|} T^{5 / 2}
$$

with,

$$
K_{\|}=\frac{1.84 \times 10^{-5}}{\ln (\mathcal{L})} \operatorname{erg~s}^{-1} \mathrm{~K}^{-1} \mathrm{~cm}^{-1},
$$

where $\ln (\mathcal{L})=29.7+\ln \left(T / 10^{6} \sqrt{n}\right)$ is the Coulomb logarithm, with $n$ the gas total number density (Spitzer 1962). The heat conduction coefficients satisfy $\kappa_{\perp} / \kappa_{\mid} \approx 10^{-16} \ll 1$ for the densities that we consider (Parker 1963; Velázquez et al. 2004; Balsara et al. 2008; Orlando et al. 2008). The value of $\boldsymbol{F}_{\mathbf{c}}$ varies between the classical flux in Eq. 111 and the saturated conduction regime (Balsara et al. 2008) which limits the heat flux to,

$$
F_{\text {sat }}=5 \phi \rho c_{\text {iso }}^{3}
$$

for very large temperature gradients $\left(\geqslant 10^{6} \mathrm{~K} \mathrm{pc}^{-1}\right)$, with $c_{\text {iso }}=$ $p / \rho$ the isothermal speed of sound and $\phi<1$ a free parameter that we set to the typical value of 0.3 (Cowie \& McKee 1977).

\subsection{Setting up the stellar wind}

We impose the stellar wind at the surface of a sphere of radius $20 \Delta z$ pc centered into the origin $O$ with wind material. Its density is,

$$
\rho_{w}=\frac{\dot{M}}{4 \pi r^{2} v_{\mathrm{w}}}
$$

where $\dot{M}$ is the star's mass-loss rate and $r$ the distance to the origin $O$. We interpolate the wind parameters from stellar evolution models of non-rotating massive stars with solar metallicity that we used for previous studies, see Paper I. Our stellar wind models are have been generated with the stellar evolution code described in Heger et al. (2005) and subsequently updated by Yoon \& Langer (2005); Petrovic et al. (2005) and Brott et al. (2011). It utilises the mass-loss prescriptions of Kudritzki et al. (1989) for the mainsequence phase of our massive stars and of de Jager et al. (1988) for the red supergiant phase. Despite of the fact that our wind models report the marginal evolution of main-sequence winds, see Paper I, they remain quasi-constant during the part of the stellar evolution that we follow. We refer the reader interested in a graphical representation of the utilised wind models to the fig 3 of Paper I, while we report the wind properties at the beginning of our simulations 
Table 1. Stellar wind parameters at the beginning of the simulations, at a time $t_{\text {start }}$ after the beginning of the zero-age main-sequences of the star. Parameter $M_{\star}\left(\right.$ in $\mathrm{M}_{\odot}$ ) is the initial mass of the star, $L_{\star}$ the stellar luminosity (in $\mathrm{L}_{\odot}$ ), $\dot{M}$ its mass loss and $v_{\mathrm{w}}$ the wind velocity, see also table 1 of Mever et al. (2016).

\begin{tabular}{cccccc}
\hline$M_{\star}\left(\mathrm{M}_{\odot}\right)$ & $t_{\text {start }}(\mathrm{Myr})$ & $\log \left(L_{\star} / \mathrm{L}_{\odot}\right)$ & $\log \left(\dot{M} / \mathrm{M}_{\odot} \mathrm{yr}^{-1}\right)$ & $v_{\mathrm{w}}\left(\mathrm{km} \mathrm{s}^{-1}\right)$ & $T_{\text {eff }}(\mathrm{K})$ \\
\hline 10 & 5.0 & 3.80 & -9.52 & 1082 & 25200 \\
20 & 3.0 & 4.74 & -7.38 & 1167 & 33900 \\
40 & 0.0 & 5.34 & -6.29 & 1451 & 42500 \\
\hline
\end{tabular}

in our Table 1 Note that our adopted values for the stellar wind velocity belong to the lower limit of the range of validity for stellar winds of OB stars (see below in Section 3.1.3.

Since we assume a spherically symmetric stellar wind density, thermal pressure and velocity profiles, we use the Parker prescription (Parker 1958) to model the magnetic field in the stellar wind. It consists of a radial component of the field,

$$
B_{r}=B_{\star}\left(\frac{R_{\star}}{r}\right)^{2},
$$

where $B_{\star}$ and $R_{\star}$ are the stellar surface magnetic field and the stellar radius, respectively, and of a toroidal component, which, in the case of a non-rotating star, this reduces to $B_{\phi}=0$. The $\propto 1 / r^{2}$ radial dependence of Eq. (16) makes the strength of the stellar magnetic field almost negligible at the wind termination shock that is typically about a few tenths of pc from the star that we study (Paper I). However, imposing a null magnetic field in the stellar wind region would let the direction of the heat flux $\boldsymbol{F}_{\mathbf{c}}$ undetermined in the region of (un)shocked wind material of the bow shock, see magnetic field unit vector $\hat{b}$ in the right-hand side of Eq. 11. Note that, given their analogous radial dependance on $r$, stellar wind and stellar magnetic field are similarly implemented into our axisymmetric simulations. In these simulations the stellar surface magnetic field is set to $B_{\star} \simeq 1.0 \mathrm{kG}$ (Donati et al.2002) at $R_{\star}=3.66 R_{\odot}$ (Brott et al. 2011) where $R_{\odot}$ is the solar radius.

\subsection{Setting up the ISM}

Our runaway stars are moving through the warm ionised phase of the ISM, i.e. we assume that they run in their own H II region inside which the gas is considered as homogeneous, laminar and fully ionised fluid. The ISM composition assumes solar metalicity (Lodders 2003), with $n_{\mathrm{H}}=0.57 \mathrm{~cm}^{-3}$ Wolfire et al. 2003) and with $T_{\text {ISM }} \approx 8000 \mathrm{~K}$, initially. The model is a moving star within an ISM at rest. We solve the equations of motion in the frame in which the star is at rest and, hence, the ISM moves with $v_{\text {ISM }}=-v_{\star}$, where $v_{\star}$ is the bulk motion of the star. The gas in the computational domain is evaluated with the cooling curve for photoionised gas described in fig. 4a of Paper I. In particular, our initial conditions neglect the possibility that a bow shock might trap the ionising front of the $\mathrm{H}$ II region (see section 2.4 of Paper I for an extended discussion of the assumptions underlying our method for modelling bow shocks from hot massive stars). Additionally, an axisymmetric magnetic field $\boldsymbol{B}=-B_{\text {ISM }} \hat{\boldsymbol{z}}$ field is imposed over the whole computational domain, with $B_{\mathrm{ISM}}>0$ its strength and $\hat{z}$ the unit vector along the $O z$ direction. Finally, our simulations trace the respective proportions of ISM gas with respect to the wind material using a passive scalar tracer according to the advection equation,

$$
\frac{\partial(\rho Q)}{\partial t}+\nabla \cdot(\boldsymbol{v} \rho Q)=0
$$

where $Q$ is a passive tracer which initial value is $Q(\boldsymbol{r})=1$ for the wind material and $Q(\boldsymbol{r})=0$ for the ISM gas, respectively.

\subsection{Simulation ranges}

We first focus on a baseline bow shock generated by an initially $20 \mathrm{M}_{\odot}$ star moving with a velocity $v_{\star}=40 \mathrm{~km} \mathrm{~s}^{-1}$ in the Galactic plane of the Milky Way whose magnetic field is assumed to be $B_{\text {ISM }}=7 \mu \mathrm{G}$ (Draine 2011). Then, we consider models with velocity $v_{\star}=20$ to $70 \mathrm{~km} \mathrm{~s}^{-1}$, explore the effects of a magnetisation of $B_{\mathrm{ISM}}=3.5 \mu \mathrm{G}$, and carry out simulations of initially 10 and $40 \mathrm{M}_{\odot}$ stars moving at velocities $v_{\star}=40$ and $70 \mathrm{~km} \mathrm{~s}^{-1}$, respectively. We investigate the effects of the ISM magnetic field carrying out a couple of additional purely hydrodynamical simulations, as comparison runs. All our simulations are started at a time about 4.5 Myr after the zero-age main-sequence phase of our stars and are run at least four crossing times $\left|z_{\max }-z_{\min }\right| / v_{\star}$ of the gas through the computational domain, such that the system reaches a steady or quasi-stationary state in the case of a stable or unstable bow shock, respectively.

We label our magneto-hydrodynamical simulations concatenating the values of the initial mass $M_{\star}$ of the moving star (in $\mathrm{M}_{\odot}$ ), its bulk motion $v_{\star}$ (in $\mathrm{km} \mathrm{s}^{-1}$ ) and the included physics "Ideal" for dissipativeless simulations, "Cool" if the model includes heating and losses by optically-thin radiative processes, "Heat" for heat conduction and "All" if cooling, heating and heat conduction are taken into account together). Finally, the labels inform about the strength of the ISM magnetic field. We distinguish our magneto-hydrodynamical runs from our previously published hydrodynamical studies (Paper I) adding the prefix "HD" and "MHD" to the simulations labels of our hydrodynamical and magnetohydrodynamical simulations, respectively. All the informations relative to our models are summarised in Table 2

\section{RESULTS AND DISCUSSION}

This section presents the magneto-hydrodynamical simulations carried out in the context of our Galactic, ionizing, runaway massive stars. We detail the effects of the included microphysics on a baseline bow shock model, we discuss the morphological differences between our hydrodynamical and magneto-hydrodynamical simulations and we consider the effects of the adopted stellar wind models. Finally, review the limitations of the model.

\subsection{Bow shock thermodynamics}

\subsubsection{Effects of the included physics: hydrodynamics}

In Fig. 1) we show the gas density field in a series of bow shock models of our initially $20 \mathrm{M}_{\odot}$ star moving with velocity $40 \mathrm{~km} \mathrm{~s}^{-1}$ through a medium of ISM background density $n_{\mathrm{H}}=0.59 \mathrm{~cm}^{-3}$ 
Table 2. Nomenclature and grid parameters used in our (magneto-)hydrodynamical simulations. The quantities $M_{\star}$ (in $M_{\odot}$ ) and $v_{\star}$ (in $\mathrm{kms}^{-1}$ ) are the initial mass of the stars and their space velocity, respectively, whereas $B_{\mathrm{ISM}}$ (in $\mu \mathrm{G}$ ) is the strength of the ISM magnetic field. Parameters $\Delta, z_{\min }$ and $R_{\max }$ are the resolution of the uniform grid (in pc cell ${ }^{-1}$ ) and the lower and upper limits of the domain along the R-axis and z-axis (in pc), respectively. The last column contains the physics included in each simulation. Heat conduction (HC) refers to isotropic thermal conduction in the case of an hydrodynamical (HD) simulation and to anisotropic thermal conduction in the case of an magneto-hydrodynamical (MHD) simulation, respectively.

\begin{tabular}{lccccccc}
\hline Model & $M_{\star}\left(M_{\odot}\right)$ & $v_{\star}\left(\mathrm{km} \mathrm{s}^{-1}\right)$ & $B_{\text {ISM }}(\mu \mathrm{G})$ & $\Delta\left(10^{-3} \mathrm{pc} \mathrm{cell}^{-1}\right)$ & $z_{\min }(\mathrm{pc})$ & $R_{\max }(\mathrm{pc})$ & Included microphysics \\
\hline HD2040Ideal & 20 & 40 & - & 8.0 & -2.0 & 8.0 & HD, adiabatic \\
HD2040Cool & 20 & 40 & - & 8.0 & -2.0 & 8.0 & HD, cooling, heating \\
HD2040Heat & 20 & 40 & - & 8.0 & -2.0 & 8.0 & HD, HC \\
HD2040All & 20 & 40 & - & 8.0 & -2.0 & 8.0 & HD, cooling, heating, HC \\
MHD2040IdealB7 & 20 & 40 & 7.0 & 8.0 & -2.0 & 8.0 & MHD \\
MHD2040CoolB7 & 20 & 40 & 7.0 & 8.0 & -2.0 & 8.0 & MHD, cooling, heating \\
MHD2040HeatB7 & 20 & 40 & 7.0 & 8.0 & -2.0 & 8.0 & MHD, HC \\
MHD2040AllB7 & 20 & 40 & 7.0 & 8.0 & -2.0 & 8.0 & MHD, cooling, heating, HC \\
MHD1040AllB7 & 10 & 40 & 7.0 & 3.0 & -2.0 & 6.0 & MHD, cooling, heating, HC \\
MHD2020AllB7 & 20 & 20 & 7.0 & 6.0 & -3.0 & 12.0 & MHD, cooling, heating, HC \\
MHD2040AllB3.5 & 20 & 40 & 3.5 & 8.0 & -2.0 & 8.0 & MHD, cooling, heating, HC \\
MHD2070AllB7 & 20 & 70 & 7.0 & 1.2 & -1.0 & 3.0 & MHD, cooling, heating, HC \\
MHD4070AllB7 & 40 & 70 & 7.0 & 1.6 & -4.0 & 16.0 & MHD, cooling, heating, HC \\
\hline
\end{tabular}

and of magnetic field strength $B_{\mathrm{ISM}}=7 \mu \mathrm{G}$. The crosses indicate the position of the moving star. The figures correspond to a time about 5 Myr after the beginning of the main-sequence phase of the star. The stellar wind and ISM properties are the same for all figures, only the included physics is different for each models (our Table 2). Left-hand panels are hydrodynamical simulations whereas right-hand panels are magneto-hydrodynamical simulations, respectively. From top to bottom, the included thermodynamic processes are adiabatic (a), take into account optically-thin radiative processes of the gas (b), heat transfers (c) or both (d). The black dotted lines are the contours $Q(\boldsymbol{r})=1 / 2$ which trace the discontinuity between the stellar wind and the ISM gas. The streamlines (a-c) and vector velocity field (d) highlight the penetration of the ISM gas into the different layers of the bow shock.

The internal structure of the bow shocks can be understood by comparing the timescales associated to the different physical processes at work. The dynamical timescale represents the time interval it takes the gas to advect through a given layer of our bow shocks, i.e. the region of shocked ISM or the layer of shocked wind. It is defined as,

$$
t_{\mathrm{dyn}}=\frac{\Delta l}{v}
$$

where $\Delta l$ is the characteristic lengthscale of the region of the bow shock measured along the $O z$ direction and where $v$ is the gas velocity in the post-shock region of the considered layers. According to the Rankine-Hugoniot relations and taking into account the non-ideal character of our model, we should have $v \simeq v_{\star} / 4$ in the shocked ISM and $v \simeq v_{\mathrm{w}} / 4$ in the post-region at the forward shock and at the reverse shock, respectively.

The cooling timescale is defined as,

$$
t_{\mathrm{cool}}=\frac{E_{\mathrm{int}}}{E_{\mathrm{int}}}=\frac{p}{(\gamma-1) \Lambda(T) n_{\mathrm{H}}^{2}},
$$

where $\dot{E_{\text {int }}}$ is the rate of change of internal energy $E_{\text {int }}$ (Orlando et al. 2005). The heat conduction timescale measures the rapidity of heat transfer into the bow shock, and is given by,

$$
t_{\text {heat }}=\frac{7 p l^{2}}{2(\gamma-1) \kappa(T) T},
$$

Table 3. Characteristics dynamical timescale $t_{\mathrm{dyn}}$, cooling timescale $t_{\mathrm{cool}}$ and thermal conduction timescale $t_{\text {heat }}$ (in Myr) measured along the $O z$ direction from our simulations of our initially $20 \mathrm{M}_{\odot}$ star moving velocity $40 \mathrm{~km} \mathrm{~s}^{-1}$ (see Fig. 17-h). We estimate the various timescales in both the post-shock region at the forward shock (FS) and the reverse shock (RS) of our bow shocks. The black hyphen indicate that the corresponding physical process is not included in the models (our Table 2 .

\begin{tabular}{lccc}
\hline Model & $t_{\text {dyn }}(\mathrm{Myr})$ & $t_{\text {cool }}(\mathrm{Myr})$ & $t_{\text {heat }}(\mathrm{Myr})$ \\
\hline HD2040Ideal (FS) & $2.5 \times 10^{-2}$ & - & - \\
HD2040Ideal (RS) & $4.7 \times 10^{-3}$ & - & - \\
HD2040Cool (FS) & $1.0 \times 10^{-2}$ & $4.5 \times 10^{-3}$ & - \\
HD2040Cool (RS) & $3.7 \times 10^{-3}$ & $3.5 \times 10^{+3}$ & - \\
HD2040Heat (FS) & $6.5 \times 10^{-2}$ & - & $1.2 \times 10^{+3}$ \\
HD2040Heat (RS) & $1.3 \times 10^{-2}$ & - & $1.2 \times 10^{-4}$ \\
HD2020All (FS) & $9.0 \times 10^{-3}$ & $5.1 \times 10^{-3}$ & $8.7 \times 10^{+5}$ \\
HD2020All (RS) & $1.1 \times 10^{-2}$ & $2.5 \times 10^{+1}$ & $4.0 \times 10^{-3}$ \\
MHD2040IdealB7 (FS) & $1.8 \times 10^{-2}$ & - & - \\
MHD2040IdealB7 (RS) & $4.3 \times 10^{-3}$ & - & - \\
MHD2040CoolB7 (FS) & $1.1 \times 10^{-1}$ & $4.3 \times 10^{-2}$ & - \\
MHD2040CoolB7 (RS) & $2.0 \times 10^{-3}$ & $1.0 \times 10^{+3}$ & - \\
MHD2040HeatB7 (FS) & $1.0 \times 10^{-2}$ & - & $1.1 \times 10^{+25}$ \\
MHD2040HeatB7 (RS) & $6.7 \times 10^{-3}$ & - & $5.5 \times 10^{+9}$ \\
MHD2040AllB7 (FS) & $3.0 \times 10^{-1}$ & $3.4 \times 10^{-3}$ & $1.4 \times 10^{+18}$ \\
MHD2040AllB7 (RS) & $2.3 \times 10^{-3}$ & $3.0 \times 10^{+4}$ & $5.4 \times 10^{+7}$ \\
\hline
\end{tabular}

where $l$ is a characteristic length of the bow shock along which heat transfers take place. Measuring the density, pressure and velocity fields in our simulations, we evaluate and compare those quantities defined in Eqs. (18)-20 at both the post-shock regions at the forward and reverse shocks. Results for both the layers of shocked wind and shocked ISM material are given in Table 3

Our hydrodynamical, dissipation-free bow shock model HD2040Ideal has a morphology governed by the gas dynamics only (Fig. 11). It has a contact discontinuity separating the outer region of cold shocked ISM from the inner region of hot shocked stellar wind, which are themselves bordered by the forward and reverse shocks, respectively. There is no advection of ISM material into the wind region (see the ISM gas streamlines in Fig. 19). The model HD2040Cool including cooling by optically-thin radiation has a considerably reduced layer of dense, shocked ISM 

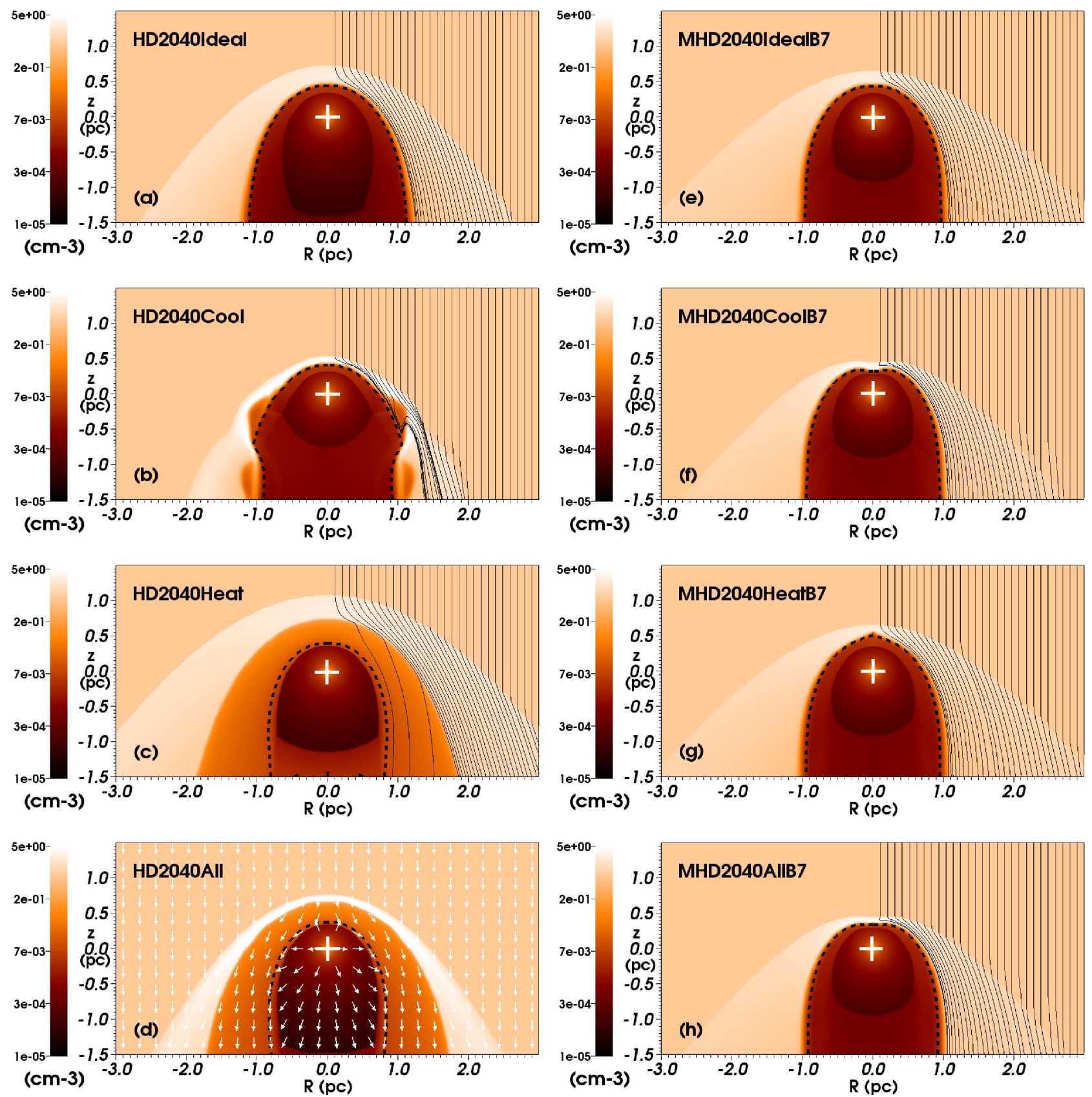

Figure 1. Changes in the morphology of a stellar wind bow shock with variation of the included physics. Figures show gas number density plotted with a density range from $10^{-5}$ to $5 \mathrm{~cm}^{-3}$ in the logarithmic scale for an initially $20 \mathrm{M}_{\odot}$ star moving with velocity $40 \mathrm{~km} \mathrm{~s}{ }^{-1}$. Left-hand panels are the hydrodynamical models whereas right-hand panels are the magneto-hydrodynamical models with $B_{\mathrm{ISM}}=7 \mu \mathrm{G}$. The first line of panels shows adiabatic (a) and ideal magneto-hydrodynamical (e) models, respectively. The second line of panels plots models with optically-thin radiative processes (b,f), the third line shows models including thermal (an-)isotropic conduction (c,g) and the last line plots models models including cooling, heating and (an-)isotropic thermal conduction $(\mathrm{d}, \mathrm{h})$. The nomenclature of the models follows our Table 2 For each figure the dotted thick line traces the material discontinuity, i.e. the interface of the wind/ISM regions, $Q(\boldsymbol{r})=1 / 2$. The right part of each figure overplots ISM flow streamlines, except panel (d) which explicitly plots the velocity field as white arrows over the whole computational domain. The crosses mark the position of the star. The R-axis represents the radial direction and the z-axis the direction of stellar motion (in pc). Only a fraction of the computational domain is shown.

gas caused by the rapid losses of internal energy $\left(t_{\mathrm{dyn}} \gg t_{\mathrm{cool}}\right.$, see timescales in our Table 3]. Its thinness favours the growth of Kelvin-Helmholtz instabilities and allows large eddies to develop in the shocked regions (Fig. 1b). The layer of hot gas is isothermal because the regular wind momentum input at the reverse shock prevents it from cooling and it therefore conserves its hot tempera- ture $\left(t_{\mathrm{cool}} \gg t_{\mathrm{dyn}}\right)$ whereas the distance between the star and the contact discontinuity,

$$
R(0)=\sqrt{\frac{\dot{M} v_{\mathrm{w}}}{4 \pi \rho_{\mathrm{ISM}} v_{\star}^{2}}},
$$


does not evolve (Wilkin 1996).

The model HD2040Heat takes into account thermal conduction which is isotropic in the case of the absence of magnetic field. The heat flux reads,

$$
\boldsymbol{F}_{\mathbf{c}}=\kappa \boldsymbol{\nabla} T
$$

and transports internal energy from the reverse shock to the contact discontinuity $\left(t_{\text {dyn }} \gg t_{\text {heat }}\right)$ which in its turn splits the dense region into a hot $\left(t_{\text {dyn }} \gg t_{\text {heat }}\right)$ and a cold layer of shocked ISM gas $\left(t_{\mathrm{dyn}} \ll t_{\text {heat }}\right)$, respectively. This modifies the penetration of ISM gas into the bow shock and causes the region of shocked wind to shrink to a narrow layer of material close to the reverse shock (Fig. 1). Not surprisingly, the model with both cooling and conduction HD2040All (Fig. 11) presents both the thermally split region of shocked ISM $\left(t_{\text {dyn }} \ll t_{\text {heat }}, t_{\text {dyn }} \gg t_{\text {cool }}, t_{\text {cool }} \ll t_{\text {heat }}\right)$ and a reduced layer of shocked wind material $\left(t_{\mathrm{dyn}} \gg t_{\text {heat }}\right.$, $\left.t_{\text {dyn }} \ll t_{\text {cool }}, t_{\text {cool }} \gg t_{\text {heat }}\right)$ that reorganises the internal structure of the bow shock together with a dense shell of cool ISM gas (see the also discussion in Paper I). For the sake of clarity Fig. 10 overplots the gas velocity fields as white arrows which illustrate the penetration of ISM gas into the hot layer of the bow shock.

\subsubsection{Effects of the included physics: magneto-hydrodynamics}

We plot in the right-hand panels of Fig. 1 the ideal magnetohydrodynamical simulation of our initially $20 \mathrm{M}_{\odot}$ star moving with $v_{\star}=40 \mathrm{~km} \mathrm{~s}^{-1}$ through a medium where the strength of the magnetic field is $B_{\text {ISM }}=7 \mu \mathrm{G}$ (e) together with models including cooling and heating by optically-thin radiation (f), anisotropic heat conduction (g) and both (h). Despite of the fact that the overall morphology of our magneto-hydrodynamical bow shock models is globally similar to the models with $B_{\mathrm{ISM}}=0 \mu \mathrm{G}$, a given number of significant changes relative to both their shape and internal structure arise. Note that in the context of our magneto-hydrodynamical models, $t_{\text {heat }}$ represents the heat transfer timescale normal to the fields lines.

Our ideal magneto-hydrodynamical model has the typical structure of a stellar wind bow shock, with a region of shocked ISM gas surrounding the one of shocked wind gas. The contact discontinuity acts as a border between the two kind of material (Fig. 1e). The model with cooling MHD2040CoolB7 has reduced but denser layer of ISM gas (Fig. 1f) due to the rapid cooling time $\left(t_{\text {cool }} \ll t_{\text {dyn }}\right)$. The magneto-hydrodynamical model with thermal conduction is similar to our model MHD2040IdealB7 since, due to his anisotropic character, heat transport are canceled across the magnetic field lines $\left(t_{\text {heat }} \ggg t_{\text {dyn }}\right)$. Note the boundary effect close to the apex along the $\mathrm{Oz}$ direction as a result of the heat conduction along the direction of the ISM magnetic field lines (Fig. 1 $\mathrm{g}$ ). Finally, our model with both processes has its dynamics governed by the cooling in the region of shocked ISM $\left(t_{\text {heat }} \ggg t_{\mathrm{dyn}}\right.$, $\left.t_{\text {heat }} \ggg t_{\text {cool }}, t_{\text {cool }} \ll t_{\text {dyn }}\right)$ and by the wind momentum in the region of shocked wind $\left(t_{\text {heat }} \ggg t_{\text {dyn }}, t_{\text {heat }} \ggg t_{\text {cool }}\right.$, $\left.t_{\text {dyn }} \ll t_{\text {cool }}\right)$.

\subsubsection{Effects of the boundary conditions: stellar wind models}

The shape of the bow shock generated around a runaway massive star in the warm phase of the ISM is a function of the respective strength of both the ISM ram pressure $\rho_{\mathrm{ISM}} v_{\star}^{2}$ and the stellar wind ram pressure $\rho_{\mathrm{w}} v_{\mathrm{w}}^{2}$, as seen in the frame of reference of the moving object (see explanations in Mohamed et al. 2012). According

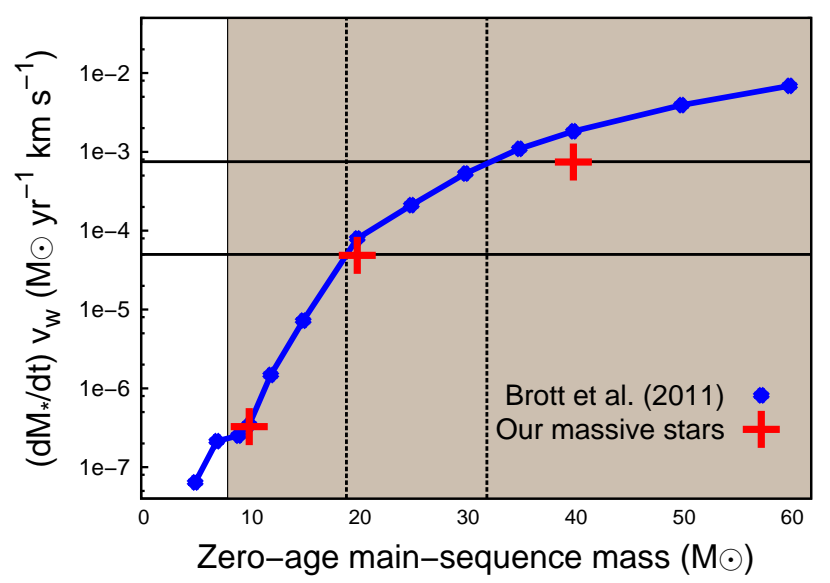

Figure 2. Comparison of the quantity $\dot{M} \mathrm{v}_{\mathrm{w}}$ between our weak-winded stars and the non-rotating Galactic models of Brott et al. (2011). The grey zone of the plot corresponds to the mass regime of massive stars $\left(M_{\star} \geqslant\right.$ $\left.8 \mathrm{M}_{\odot}\right)$. Solid and dotted lines are lines of constant $\dot{M} v_{w}$ and $M_{\star}$, respectively.

to Eq. 15, $\rho_{w}=\dot{M} / 4 \pi r^{2} v_{\mathrm{w}}$ which implies that $\rho_{\mathrm{w}} v_{\mathrm{w}}^{2} \propto \dot{M} v_{\mathrm{w}}$. In other words, in a given ambient medium and at a given peculiar velocity, the governing quantity in the shaping of such bow shock is $\dot{M} \mathrm{v}_{\mathrm{w}}$ and its stand-off distance $R(0)$ goes as $\sqrt{\dot{M} v_{w}}$, see Eq. 21. Nevertheless, if the production of stellar evolution models depends on specific prescriptions relative to $\dot{M}$ that are consistently used through the calculations (in our case the recipe of Kudritzki et al. 1989), the estimate of the wind velocity is posterior to the calculation of the stellar structure and it does not influence $\dot{M}, T_{\text {eff }}$ or $L_{\star}$.

The manner to calculate $\mathrm{v}_{\mathrm{w}}$ is not unique (Castor et al. 1975; Kudritzki et al. 1989; Kudritzki \& Puls 2000; Eldridge et al. 2006) and it can also be assumed to characteristic values for the concerned stars (Comerón \& Kaper 1998; van Marle et al. 2014, 2015; Acreman et al. 2016). In our study, the wind velocities are in the lower limit of the range of validity for the main-sequence massive stars that we consider, nonetheless, they still remain within the order of magnitude of, e.g. late O stars (Martins et al. 2007) or weak-winded stars (Comerón \& Kaper 1998). Furthermore, the evolution of massive stars are governed by physical mechanisms strongly influencing their feedback such as the presence of lowmass companions (Sana et al. 2012), which are neglected in our stellar evolution models. Produced before their zero-age mainsequence phase, e.g. by fragmentation of the accretion disk that surrounds massive protostars (Meyer et al.2016), those dwarf stars entirely modify the evolution of massive stars and consequently affect their wind properties (de Mink et al. 2007, 2009; Paxton et al. 2011; Marchant et al. 2016).

Using wind velocities faster by a factor $\alpha$ would enlarge the bow shocks by a factor $\sqrt{\alpha}$ and, eventually, in the hydrodynamical case, favorise the growth of instabilities (cf. Fig. 1b). However, the results of our numerical study would be similar in the sense that the presence of the field essentially stabilises the nebulae and inhibits the effects of heat conduction (cf. Fig. 11,h), reduces their size (Section 3.2.1 and modifies, e.g. their infrared emission accordingly (see Section 4.3. In Fig. 2 we compare our values of $\dot{M} v_{w}$ (Table 1) with the non-rotating stellar evolutionary models published in Brott et al. (2011). We conclude that the bow shocks generated with our initially 10,20 and $40 \mathrm{M}_{\odot}$ weak-winded stellar 
models correspond to nebulae produced by initially $\approx 10, \approx 18$ and $\approx 32 \mathrm{M}_{\odot}$ standard massive stars at Galactic metallicity, respectively. Therefore, our models have full validity for this study of magnetized bow shock nebulae, albeit of lower zero-age mainsequence mass in the case of our heaviest runaway star.

\subsection{Hydrodynamics versus magneto-hydrodynamics}

\subsubsection{The effects of the magnetic pressure}

The ISM magnetic pressure, proportional to $\vec{B}_{\text {ISM }}^{2}$, dynamically compresses the region of shocked ISM gas such that the density in the post-shock region at the forward shock slightly increases. Similarly, the shape of the bow shock's wings of shocked ISM are displaced sidewards compare to our model with $B_{\text {ISM }}=0 \mu \mathrm{G}$ (Fig. 1 1 ,e). The size of the layer of ISM gas diminishes along the direction of motion of the moving star and the position of the termination shock sets at a distance from the star where the wind ram pressure equals the ISM total pressure decreases as measured along the $O z$ axis. The effects of the cooling is standard in the sense that it makes the region of shocked ISM thinner and denser, i.e. the position of the forward shock decreases, together with the bow shock volume. The effects of heat conduction are canceled $\left(t_{\mathrm{dyn}} \ll t_{\text {heat }}\right)$ in the direction perpendicular to the field lines, i.e. in the direction perpendicular to the streamline collinear to both the reverse shock and the contact discontinuity.

\subsubsection{Stagnation point morphology and discussion in the context of plasma physics studies}

The topology at the apex of our magneto-hydrodynamical bow shock (Fig. 1h) is different from the traditional single-front bow shock morphology (Fig. 1 d). This can be discussed at the light of plasma physics studies (de Sterck et al. 1998; de Sterck \& Poedts 1999). These works explore the formation of exotic shocks and discontinuities that affect the particularly dimpled apex of bow shocks generated by field-aligned flows around a conducting cylinder (de Sterck et al. 1998). They extended this result to bow shocks produced around a conducting sphere and showed that the inflow parameter space leading to such structures is similar to plasma $\beta$ and Alfvénic Mach number values allowing the formation of socalled switch-on shocks (de Sterck \& Poedts 1999).

Switch-on shocks are allowed when plasma $\beta$ of the inflowing material, i.e. the ratio of the gas and magnetic pressures, which read,

$$
\beta=\frac{8 \pi n k_{\mathrm{B}} T}{\boldsymbol{B}_{\mathrm{ISM}} \cdot \boldsymbol{B}_{\mathrm{ISM}}},
$$

and its Alfvénic Mach number,

$$
M_{\mathrm{A}}=\frac{v}{v_{\mathrm{A}}},
$$

where,

$$
v_{\mathrm{A}}=\frac{\left|\boldsymbol{B}_{\mathrm{ISM}}\right|}{\sqrt{4 \pi n m_{\mathrm{H}}}},
$$

is the Alfvénic velocity, satisfy some particular conditions. Note that in Eq. 24) the velocities are taken along the shock normal. On the one hand, the plasma beta must be such that,

$$
\beta<\frac{2}{\gamma}
$$

whereas on the other hand, the Alfvénic Mach number verifies the
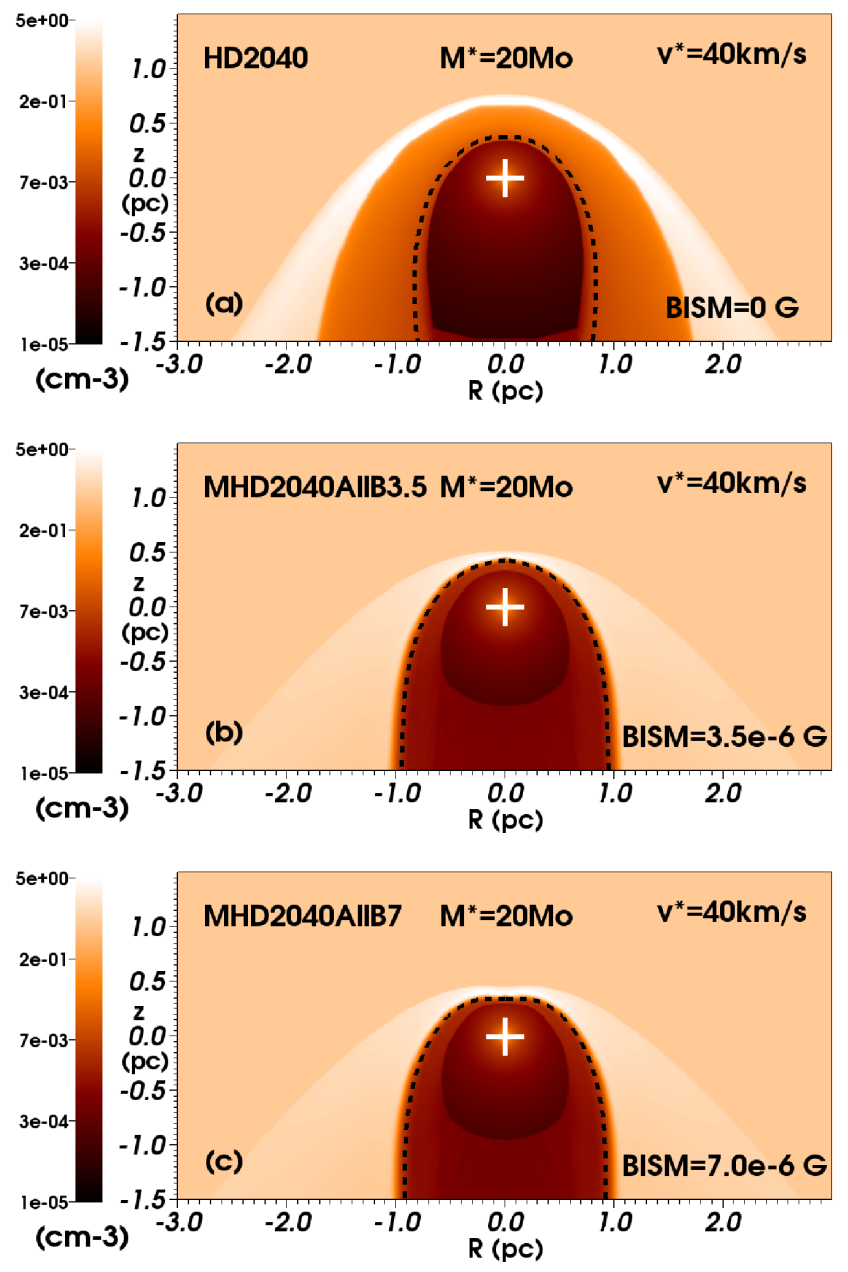

Figure 3. Models of stellar wind bow shocks of our initially $20 \mathrm{M}_{\odot}$ star moving with velocity $v_{\star}=40 \mathrm{~km} \mathrm{~s}^{-1}$ represented as a function of its ISM magnetic field strength, with $B_{\mathrm{ISM}}=0$ (a), 3.5 (b) and $7.0 \mu \mathrm{G}$ (c).

following order relation,

$$
1<M_{\mathrm{A}}<\sqrt{\frac{\gamma(1-\beta)+1}{\gamma-1}},
$$

where $\gamma$ is the adiabatic index, see Eq. 1 in Pogorelov \& Matsuda (2000). Numbers from our simulations indicate that the ISM thermal pressure $n_{\mathrm{ISM}} k_{\mathrm{B}} T_{\mathrm{ISM}} \approx 8.62 \times 10^{-13}$ dynes $^{-2}$, therefore we find $\beta>2 / \gamma \approx 1.2$ for $B_{\mathrm{ISM}} \leqslant 3.5 \mu \mathrm{G}$ (see bow shocks with normal morphologies in Fig. 3a,b) but $\beta \approx 0.44<2 / \gamma \approx 1.2$ for $B_{\text {ISM }}=7 \mu \mathrm{G}$ (see dimpled bow shock in Fig. 3r). The Alfvénic Mach number $M_{\mathrm{A}}=v / v_{\mathrm{A}} \approx 40.0 \mathrm{~km} \mathrm{~s}^{-1} / 17.2 \mathrm{~km} \mathrm{~s}^{-1} \approx 2.33$ which is outside the range $1<M_{\mathrm{A}}<((\gamma(1-\beta)+1) /(\gamma-$ $1))^{1 / 2} \approx 1.70$. Similarly, the model with $v_{\star}=70 \mathrm{~km} \mathrm{~s}^{-1}$ is such that $M_{\mathrm{A}}>((\gamma(1-\beta)+1) /(\gamma-1))^{1 / 2}$ whereas our slower model with $v_{\star}=20 \mathrm{~km} \mathrm{~s}^{-1}$ gives $1<M_{\mathrm{A}} \approx 1.16<$ $((\gamma(1-\beta)+1) /(\gamma-1))^{1 / 2}$, which is inside the range in Eq. (27). We conclude that the upstream ISM conditions in our magnetohydrodynamical simulations producing dimpled bow shocks have values consistent with the existence of switch-on shocks, see also sketch of the $\left(\beta, M_{\mathrm{A}}\right)$ plane in Fig. 3 of de Sterck \& Poedts (1999).

However, we can not affirm that the dimpled apex topology 
of our magneto-hydrodynamical bow shocks models is of origin similar to the ones in de Sterck et al. (1998); de Sterck \& Poedts (1999). Only their particular concave-inward form that differs from the classical shape of hydrodynamical bow shocks (Fig. 11) authorizes a comparison between the two studies. Nevertheless, we notice that our bow shocks are also generated in an ambient medium in which the plasma beta and the Alfvénic Mach number have parameter values consistent with the formation of switchon shocks, which has been showed to be similar to the parameter values producing dimpled bow shocks around charged obstacles (see de Sterck et al. 1998; de Sterck \& Poedts 1999, and references therein). Additional investigations, left for future studies, are required to assess the question of the exact nature the various discontinuities affecting magneto-hydrodynamical bow shocks of OB stars.

\subsubsection{Effects of the magnetic field strength}

Fig. 3 is similar to Fig. 1 and displays the effects of the ISM magnetic field strength $B_{\text {ISM }}=0$ (a), 3.5 (b) and $7.0 \mu \mathrm{G}$ (c) on the shape of the bow shocks produced by our initially $20 \mathrm{M}_{\odot}$ star moving with velocity $v_{\star}=40 \mathrm{~km} \mathrm{~s}^{-1}$. In Fig. 12 we show density (solid lines) and temperature (dotted lines) profiles from our hydrodynamical simulation (thick blue lines) and magnetohydrodynamical model (thin red lines) of the bow shocks in Fig. 3 The profiles are taken along the symmetry axis of the computational domain. The global structure of the bow shock is similar for both simulations, i.e. it consists of a hot bubble $\left(T \approx 10^{7} \mathrm{~K}\right)$ surrounded by a shell of dense $\left(n \approx 10 \mathrm{~cm}^{-3}\right)$ shocked ISM gas. The profiles in Fig. 12 highlights the progressive compression of the bow shocks by the the ISM total pressure which magnetic component increases as $B_{\text {ISM }}$ is larger. Several mechanisms at work might be responsible for such discrepancy:

(i) The magnetic pressure in the ISM. If one neglects the thermal pressures $n k_{\mathrm{B}} T$ in both the supersonic stellar wind and the inflowing ISM, and omits the magnetic pressure $\propto B_{\star}^{2} \propto r^{-4}$ at the wind termination shock, then the pressure balance between ISM and stellar wind gas reads,

$$
\rho_{\mathrm{w}} v_{\mathrm{w}}^{2}=\rho_{\mathrm{ISM}} v_{\star}^{2}+\frac{B_{\mathrm{ISM}}^{2}}{8 \pi},
$$

from which one can derive the bow shock stand-off distance in a planar-aligned field bow shock,

$$
R(0)=\left(\frac{2 \dot{M} v_{\mathrm{w}}}{B_{\mathrm{ISM}}^{2}+8 \pi \rho_{\mathrm{ISM}} v_{\star}^{2}}\right)^{1 / 2},
$$

that is slightly smaller from the one derived in a purely hydrodynamical context (Wilkin 1996).

(ii) The cooling by optically-thin radiative processes. Changes in the density at the post-shock region at the forward shock influence the temperature in the shocked ISM gas, which in their turn modify the cooling rate of the gas, itself affecting its thermal pressure. This results in an increase of the density of the shell of ISM gas but also a decrease of the temperature in the hot region of shocked stellar wind material that shrinks in order to maintain its total pressure equal to $\rho_{\text {ISM }} v_{\star}^{2}+B_{\text {ISM }}^{2} / 8 \pi$.

(iii) The magnetic field field lines inside the bow shock. The compression of the layer of shocked ISM gas modifies the arrangement of the field lines in the post-shock region at the forward shock. Thus, the term $B_{\text {ISM }}^{2} / 8 \pi$ corresponding to the magnetic pressure increases and modifies the effects of radiative cooling in the simulations (see above).

(iv) Symmetry effects. The solution may also be affected by the intrinsic two-dimensional nature of our simulations, which may develop numerical artifices close to the symmetry axis. In the case of magneto-hydrodynamical simulations of objects moving supersonically along the direction of the ISM magnetic field, such effects are more complex than a simple accumulation of material at the apex of the nebula, but might present artificial shocks, see also Section 3.4

Appreciating in detail which of the above cited processes dominates the solution would require three-dimensional numerical simulations which are beyond the scope of this work. Moreover, establishing an analytic theory of the position of the contact discontinuity of a magnetized bow shock is a non-trivial task since the thin-shell limit (Wilkin 1996) is not applicable. In particular, the hot bubble loses about three quarter of its size along the $O z$ direction when the ISM magnetic field strength increases up to $B_{\text {ISM }}=7 \mu \mathrm{G}$ (Fig. 3h,c). This modifies the volume of hot shocked ISM gas advected thanks to heat transfers towards the inner part of the bow shock of our model HD2040All, reducing it to a narrow layer made of shocked wind material since anisotropic thermal conduction forbids the penetration of ISM gas in the hot region. The effects of the ISM magnetisation on our optical and infrared bow shocks' emission properties are further discussed in Section 4

All of our magneto-hydrodynamical simulations have a stable density field (Fig.11,f,g,h). The simulations with cooling but without heat transfer (Fig. 13) show that the presence of the magnetic field inhibits the growth of Kelvin-Helmholtz instabilities (Fig.11) that typically develops within the contact discontinuity of the bow shocks because they are the interface of two plasma moving in opposite directions (Comerón \& Kaper 1998; van Marle et al. 2007, Paper I). The solution does not change performing the simulation MHD2040AllB7 at double and quadruple spatial resolution, and conclude that our results are consistent with both numerical studies devoted to the growth and saturation of these instabilities in the presence of a planar magnetic field (see, e.g. Keppens et al. 1999) and with results obtained for slow-winded, cool runaway stars moving in a planar-aligned magnetic field (van Marle et al. 2014). Note that detailed numerical studies demonstrating the suppression of shear instabilities by the presence of a background magnetic field also exist in the context of jets from protostars Viallet \& Baty 2007).

\subsection{Effects of the star's bulk motion}

Fig. 5 is similar to Fig. 3 and plots a grid of density field of our initially $20 \mathrm{M}_{\odot}$ star moving with velocity $v_{\star}=20$ (a), 40 (b), $70 \mathrm{~km} \mathrm{~s}^{-1}$ (c). The scaling effect of the bulk motion of the star on the bow shocks morphology is similar to our hydrodynamical study (Paper I). At a given strength of the ISM magnetic field, the compression of the forward shock increases as the spatial motion of the star increases because the ambient medium ram pressure is larger. The relative thickness of the layers of ISM and wind behaves similarly as described in Paper I. Our model with $v_{\star}=20 \mathrm{~km} \mathrm{~s}^{-1}$ has a layer of shocked ISM larger than the layer of shocked wind because the relatively small ISM ram pressure induces a weak forward shock (Fig. 5a). The shell of shocked ISM is thinner in our simulation with $v_{\star}=70 \mathrm{~km} \mathrm{~s}^{-1}$ because the strong forward shock has a high post-shock temperature which allows an efficient cooling of the plasma (Fig. 55).

The density field in our models with ISM inflow velocity sim- 


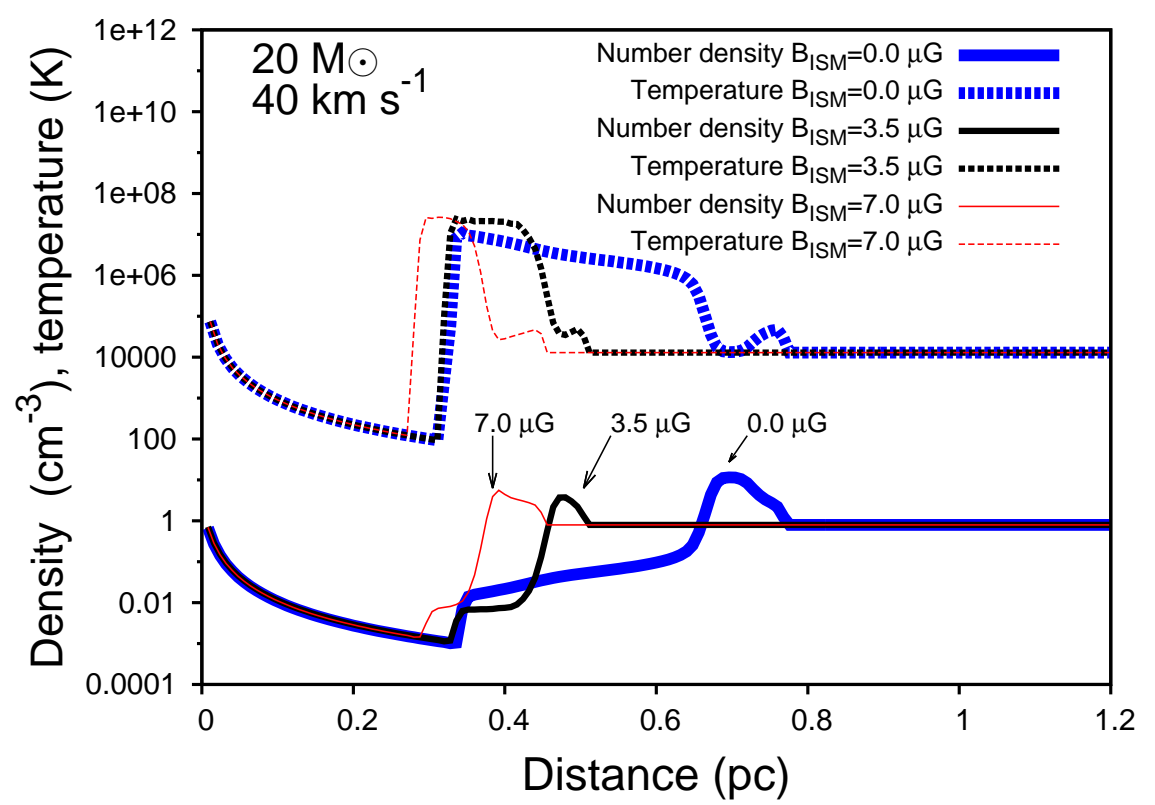

Figure 4. Number density (solid lines, in $\mathrm{cm}^{-3}$ ) and temperature (dotted lines, in $\mathrm{K}$ ) profiles in our hydrodynamical (thick blue lines) and magnetohydrodynamical (thin red lines) bow shocks models of an initial $20 \mathrm{M}_{\odot}$ star moving with velocity $v_{\star}=40 \mathrm{~km} \mathrm{~s}^{-1}$. The profiles are measured along the symmetry axis $\mathrm{Oz}$.

ilar to the Alfvénic speed $\left(v_{\star}=20 \simeq v_{\mathrm{A}} \approx 17.2 \mathrm{~km} \mathrm{~s}^{-1}\right)$ has the dimpled shape of its apex of the bow shock (Fig. 5 $\mathrm{k}$ ). The model with $v_{\star}=70 \mathrm{~km} \mathrm{~s}^{-1}$ has inflow ISM velocity larger than the Alfvénic speed and presents the classical single-front morphology (Fig. 5k) typically produced by stellar wind bow shocks (Brighenti \& D’Ercole 1995b. a; Comerón \& Kaper 1998; Meyer et al. 2016). A similar effect of the Alfvénic speed is discussed in, e.g. fig.4 of de Sterck \& Poedts (1999). Again, exploring in detail whether the formation mechanisms of our dimpled bow shocks is identical to the ones obtained in calculations of bow shock flow over a conducting sphere is far beyond the scope of this work. Note the absence of instabilities in our magneto-hydrodynamical bow shocks simulations compare to our hydrodynamical models.

\subsection{Model limitation}

First and above, our models suffer from their two-dimensional nature. If carrying out axi-symmetric models is advantageous in order to decrease the amount of computational ressources necessary to perform the simulations, however, it forbids the bow shocks from generating a structure which apex would be totally unaffected by symmetry-axis related phenomenons, common in this case of calculations (Meyer et al. 2016). This prevents our simulations from being able to assess, e.g. the question of the relation between the seeds of the non-linear thin-shell instability at the tip of the structure and the growth of Kelvin-Helmholtz instabilities occuring later in the wings of the bow shocks. Only full 3D models of the same bow shocks could fix such problems and allow us to further discuss in detail the instability of bow shocks from OB stars. We refer the reader to van Marle et al. (2015) for a discussion of the dimensiondependence of numerical solutions concerning the interaction of magnetic fields with hydrodynamical instabilities.

In particular, the selection of admissible shocks which is generally treated using artificial viscosity in purely hydrodynamical simulations is more complex in our magneto-hydrodynamical context (see discussion in Pogorelov \& Matsuda 2000). This can lead to additional fragilities of the solution, especially close to the symmetry axis of our cylindrically-symmetric models. Although the stability of these kinds of shocks is still under debate (de Sterck \& Poedts 2000, 2001), we will try to address these issues in future three-dimensional simulations. Moreover, such models would (i) allow us to explore the effects of a non-aligned ISM magnetic field on the morphology of the bow shocks and (ii) will make subsequent radiative transfer calculations meaningful, e.g. considering polarization maps using full anisotropic scattering of the photons on the dust particles in the bow shocks. The space of parameters investigated in our study is also limited, especially in terms of the explored range of space velocity $v_{\star}$ and ISM density $n_{\text {ISM }}$ and will be extended in a follow-up project. Finally, other physical processes such as the presence of a surrounding H II region or the intrinsic viscous, granulous and turbulent character of the ISM are also neglected and deserve additional investigations.

\section{COMPARISON WITH OBSERVATIONS AND IMPLICATIONS OF OUR RESULTS}

In this section, we extract observables from our simulations, compare them to observations and discuss their astrophysical implications. We first recall the used post-processing methods and then compare the emission by optically-thin radiation of our magnetohydrodynamical bow shocks with hydrodynamical models of the same star moving at the same velocity. Given the high temperature generated by collisional heating (Fig.6), we particularly focus on the $\mathrm{H} \alpha$ and [OIII] $\lambda 5007$ optical emission. Moreover, stellar wind bow shocks from massive stars have been first detected at these spectral lines and hence constitute a natural observable. We complete our analysis with infrared radiative transfer calculations and comment the observability of our bow shock nebulae. Last, we discuss our findings in the context of the runaway massive star $\zeta$ Ophiuchi. 
Table 4. Maximum optical surface brightness of our magneto-hydrodynamical simulations with $B_{\text {ISM }}=7 \mu G$. The second and third columns are the quantities $\Sigma_{[\mathrm{H} \alpha]}^{\max }$ and $\Sigma_{[\mathrm{OIII}]}^{\max }$ representing the maximum projected emission in [OIII] $\lambda 5007$ and $\mathrm{H} \alpha$ (in $\mathrm{erg} \mathrm{cm}^{-2} \mathrm{~s}^{-1} \operatorname{arcsec}^{-2}$ ), respectively. Models consisting of the hydrodynamical counterpart of our bow shocks models have their labels in italic in the first column (see description in Table 1 in Paper I). The surface brightnesses are measured along the direction of motion of the star at the apex of our bow shocks, close to the symmetry axis $O z$.

\begin{tabular}{cccc}
\hline Model & $\Sigma_{[\mathrm{H} \alpha]}^{\max }\left(\mathrm{erg} \mathrm{cm}^{-2} \mathrm{~s}^{-1} \operatorname{arcsec}^{-2}\right)$ & $\Sigma_{[\mathrm{OIII}]}^{\max }\left(\mathrm{erg} \mathrm{cm}^{-2} \mathrm{~s}^{-1} \operatorname{arcsec}^{-2}\right)$ & $\Sigma_{[\mathrm{OIII}]}^{\max } / \Sigma_{[\mathrm{H \alpha} \alpha]}^{\max }$ \\
\hline MHD1040AllB7 & $2.5 \times 10^{-19}$ & $7.0 \times 10^{-18}$ & 28.0 \\
MS1040 & $1.0 \times 10^{-18}$ & $2.5 \times 10^{-17}$ & 25.0 \\
MHD2020AllB7 & $1.7 \times 10^{-17}$ & $6.8 \times 10^{-17}$ & 4.0 \\
MS2020 & $6.0 \times 10^{-17}$ & $7.2 \times 10^{-17}$ & 1.2 \\
MHD2040AllB7 & $2.9 \times 10^{-17}$ & $1.6 \times 10^{-16}$ & 5.5 \\
MHD2040AllB3.5 & $1.0 \times 10^{-16}$ & $3.2 \times 10^{-16}$ & 3.2 \\
MS2040 & $1.2 \times 10^{-16}$ & $2.5 \times 10^{-16}$ & 2.1 \\
MHD2070AllB7 & $8.0 \times 10^{-18}$ & $2.0 \times 10^{-16}$ & 25.0 \\
MS2070 & $1.5 \times 10^{-16}$ & $8.5 \times 10^{-16}$ & 5.7 \\
MHD4070AllB7 & $1.2 \times 10^{-17}$ & $5.5 \times 10^{-16}$ & 45.8 \\
MS4070 & $4.0 \times 10^{-16}$ & $1.0 \times 10^{-15}$ & 2.5 \\
\hline
\end{tabular}

\subsection{Post-processing methods}

Fig. 7 plots the projected optical emission of our model of an initially $20 \mathrm{M}_{\odot}$ star moving at $40 \mathrm{~km} \mathrm{~s}^{-1}$ in $\mathrm{H} \alpha$ (a) and [OIII] $\lambda 5007$ (b) in $\operatorname{erg~s}^{-1} \mathrm{~cm}^{-2} \operatorname{arcsec}^{-2}$. Left-hand part of the panels correspond to the star moving into an ISM with no background magnetic field (hydrodynamical model MS2040, Paper I) whereas right-hand parts correspond to $B_{\mathrm{ISM}}=7 \mu \mathrm{G}$ (magnetohydrodynamical model MHD2040AllB7). We take into account the rotational symmetry about $R=0$ of our models and integrate the emission rate assuming that our bow shocks lay in the plane of the sky, i.e. the star moves perpendicular to the observers lineof-sight. The spectral lines emission coefficients are evaluated using the prescriptions for optical spectral line emission from Dopita (1973) and Osterbrock \& Bochkarev (1989), which read,

$$
j_{[\mathrm{H} \alpha]}(T) \approx 1.21 \times 10^{-22} T^{-0.9} n_{\mathrm{p}}^{2} \mathrm{erg} \mathrm{s}^{-1} \mathrm{~cm}^{-3} \mathrm{sr}^{-1},
$$

where $n_{\mathrm{p}}$ is the number of proton in the plasma, and,

$$
j_{[\mathrm{OIII}]}(T) \approx 3.23 \times 10^{-21} \frac{e^{-\frac{28737}{T}}}{4 \pi \sqrt{T}} n_{\mathrm{p}}^{2} \mathrm{erg} \mathrm{s}^{-1} \mathrm{~cm}^{-3} \mathrm{sr}^{-1} \text {, }
$$

for the $\mathrm{H} \alpha$ and [OIII] $\lambda 5007$ spectral lines, respectively. Additionally, we assume solar oxygen abundances (Lodders 2003) and cease to consider the oxygen as triply ionised at temperatures larger than $10^{6} \mathrm{~K}$ (cf. Cox et al. 1991).

The bow shocks luminosities $L$ are estimated integrating the emission rate,

$$
L=2 \pi \iint_{\mathcal{D}} \Lambda(T) n_{\mathrm{H}}^{2} R d R d z
$$

where $\mathcal{D}$ represents its volume in the $z>0$ part of the computational domain (Mohamed et al. 2012, Paper I). Similarly, we calulate the momentum deposited by the bow shock by subtracting the stellar motion from the ISM gas velocity field. We compute $L_{\mathrm{H} \alpha}$ and $L_{[\mathrm{OIII}]}$, the bow shocks luminosity in [OIII] $\lambda 5007$ and $\mathrm{H} \alpha$, respectively. Furthermore, we discriminate the total bow shock luminosity $L_{\text {total }}$ from the shocked wind emission $L_{\text {wind }}$. For distinguishing the two kind of material, we make use of a passive scalar $Q$ that is advected with the gas. We estimate the overall $\mathrm{X}$ rays luminosity $L_{\mathrm{X}}$ with emission coefficients generated with the XSPEC program (Arnaud 1996) with solar metalicity and chemical abundances from Asplund et al. (2009). Moreover, the total infrared emission $L_{\mathrm{IR}}$ is estimated as a fraction of the starlight bolo- metric flux $L_{\star}$ Brott et al. 2011) intercepted by the ISM silicate dust grains in the bow shock,

$$
\Gamma_{\star}^{\mathrm{dust}}=\frac{L_{\star}}{4 \pi d^{2}} n_{\mathrm{d}} \sigma_{\mathrm{d}}(1-A) \mathrm{erg} \mathrm{s}^{-1} \mathrm{~cm}^{-3},
$$

plus the collisional heating,

$$
\Gamma_{\text {coll,photo }}^{\text {dust }}(T)=\frac{2^{5 / 2} f Q n n_{\mathrm{d}} \sigma_{\mathrm{d}}}{\sqrt{\pi m_{\mathrm{p}}}}\left(k_{\mathrm{B}} T\right)^{3 / 2} \mathrm{erg} \mathrm{s}^{-1} \mathrm{~cm}^{-3},
$$

where $a=5.0 \mathrm{~nm}$ is the dust grains radius,

$$
\sigma_{\mathrm{d}}=\pi a^{2} \mathrm{~cm}^{2}
$$

is their geometrical cross-section, $d$ their distance from the star and $A=1 / 2$ their Albedo. Additionally, $n_{\mathrm{d}}$ is the dust number density whereas $Q \simeq 1$ represents the grains electrical properties. More details regarding to the estimate of the bow shock infrared luminosity are given in Appendix B of Paper I.

Last, infrared images are computed performing dust continuum calculations against dust opacity for the bow shock generated by our $20 \mathrm{M}_{\odot}$ star moving with velocity $40 \mathrm{~km} \mathrm{~s}^{-1}$, using the radiative transfer code RADMC-3 $\mathrm{D}^{2}$ (Dullemond 2012). We map the dust mass density fields in our models onto a uniform spherical grid $\left[0 ; R_{\mathrm{sph}}\right] \times\left[0 ; \theta_{\max }\right]$, where $R_{\mathrm{sph}}=\left(R_{\max }^{2}+z_{\max }^{2}\right)^{1 / 2}$ and $\theta_{\max }=180^{\circ}$. We assume a dust-to-gas mass ratio of $1 / 200$. The dust density field is computed with the help of the passive scalar tracer $Q$ that allows us to separate the dust-free stellar wind of our hot OB stars with respect to the dust-enriched regions of the bow shock, made of shocked ISM gas. Additionally, we exclude the regions of ISM material that are strongly heated by the shocks or by electronic thermal conduction (Paper I), and which are defined as much hotter than about a few $10^{4} \mathrm{~K}$. RADMC-3D then self-consistently determines the dust temperature using the MonteCarlo method of Biorkman \& Wood (2001) and Lucy (1999) that we use as input to the calculations of our synthetic observations.

The code solves the transfer equation by ray-tracing photons packages from the stellar atmosphere that we model as a black body point source of temperature $T_{\text {eff }}$ (see our Table 1) that is located at the origin of the spherical grid. The dust is assumed to be composed of silicates (Draine \& Lee 1984) of mass density $3.3 \mathrm{~g} \mathrm{~cm}^{-3}$ that follow the canonical power-law distri-

2 http://www.ita.uni-heidelberg.de/ dullemond/software/radmc-3d/ 

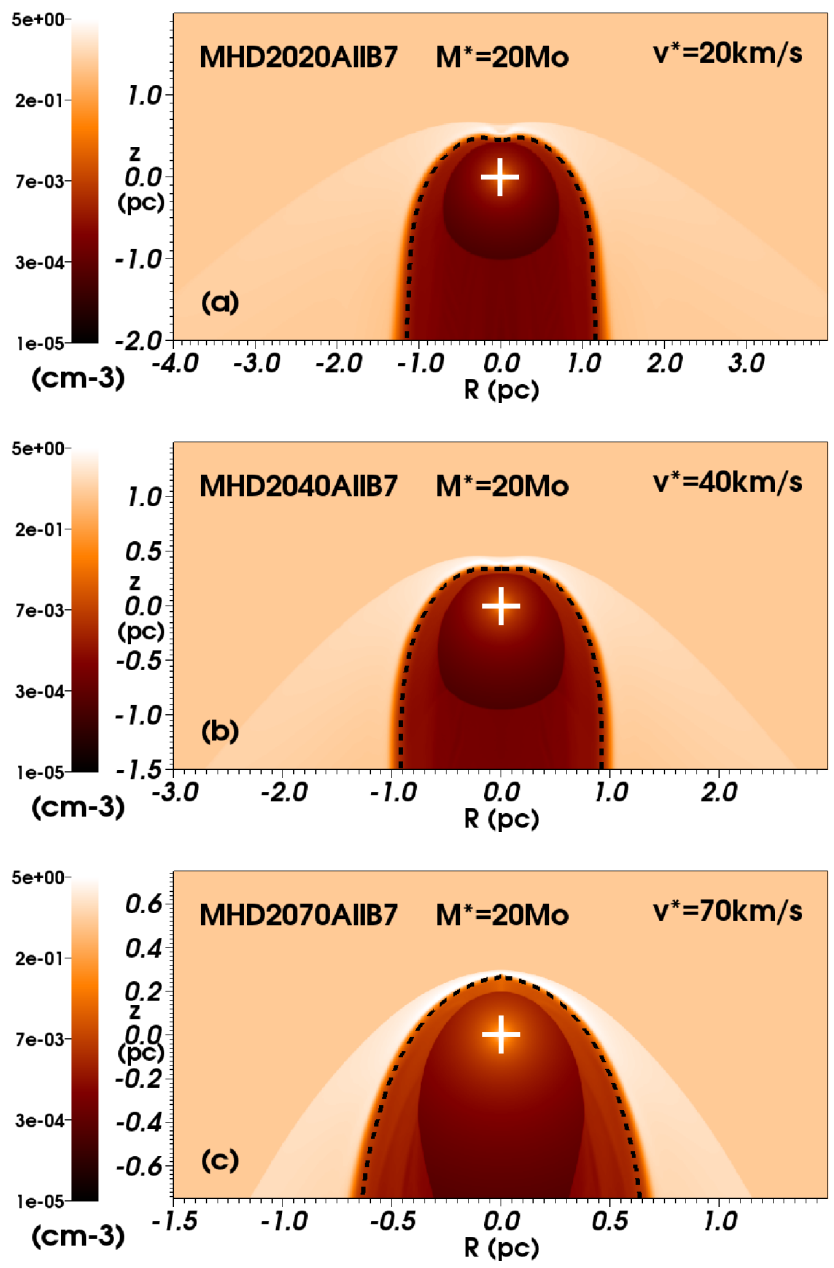

Figure 5. Grid of stellar wind bow shocks from our initially $20 \mathrm{M}_{\odot}$ star represented as a function of its space velocity with respect to the ISM, with velocity $v_{\star}=20$ (a), 40 (b) and $70 \mathrm{~km} \mathrm{~s}^{-1}$ (c). The nomenclature of the models follows Table 2 The bow shocks are shown at about $5 \mathrm{Myr}$ after the beginning of the main-sequence phase of the central star's evolution. All our MHD models assume a strength of the ISM magnetic field $B_{\mathrm{ISM}}=$ $7 \mu \mathrm{G}$. The gas number density is shown with a density range from $10^{-5}$ to $5.0 \mathrm{~cm}^{-3}$ in the logarithmic scale. The crosses mark the position of the star. The solid black contour traces the boundary between wind and ISM material $Q_{1}(\boldsymbol{r})=1 / 2$. The $R$-axis represents the radial direction and the $z$-axis the direction of stellar motion (in pc). Only part of the computational domain is shown in the figures.

bution $n(a) \propto a^{-q}$ with $q=-3.3$ (Mathis et al. 1977) and where $a_{\min }=0.005 \mu \mathrm{m}$ and $a_{\max }=0.25 \mu \mathrm{m}$ the minimal and maximal dust sizes van Marle et al. 2011). We generate the corresponding RADMC-3D input files containing the dust scattering $\kappa_{\text {scat }}$ and absorption $\kappa_{\text {abs }}$ opacities such that the total opacity $\kappa_{\text {tot }}=\kappa_{\text {scat }}+\kappa_{\text {abs }}$ (see Fig. 9 a) on the basis of a run of the Mie code of Bohren and Huffman (Bohren \& Huffman 1983) which is available as a module of the HYPERION 3 package (Robitaille 2011). Our radiative transfer calculations produces spectral energy distributions (SEDs) and isophotal images of the bow shocks at a desired wavelength, which we choose to be $\lambda=24$

\footnotetext{
${ }^{3}$ http://www.hyperion-rt.org/
}

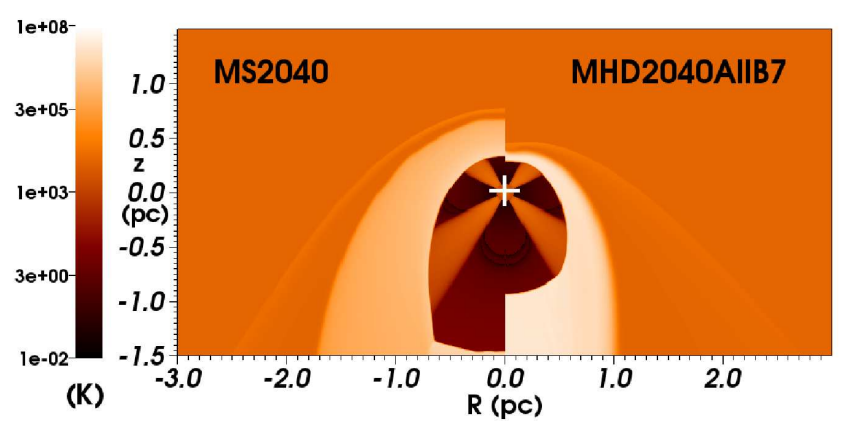

Figure 6. Temperature field (in K) in the models MS2040 and MHD2040AllB7. The cross-like structure in the central region of freelyexpanding stellar wind is a boundary effects caused by the pressure
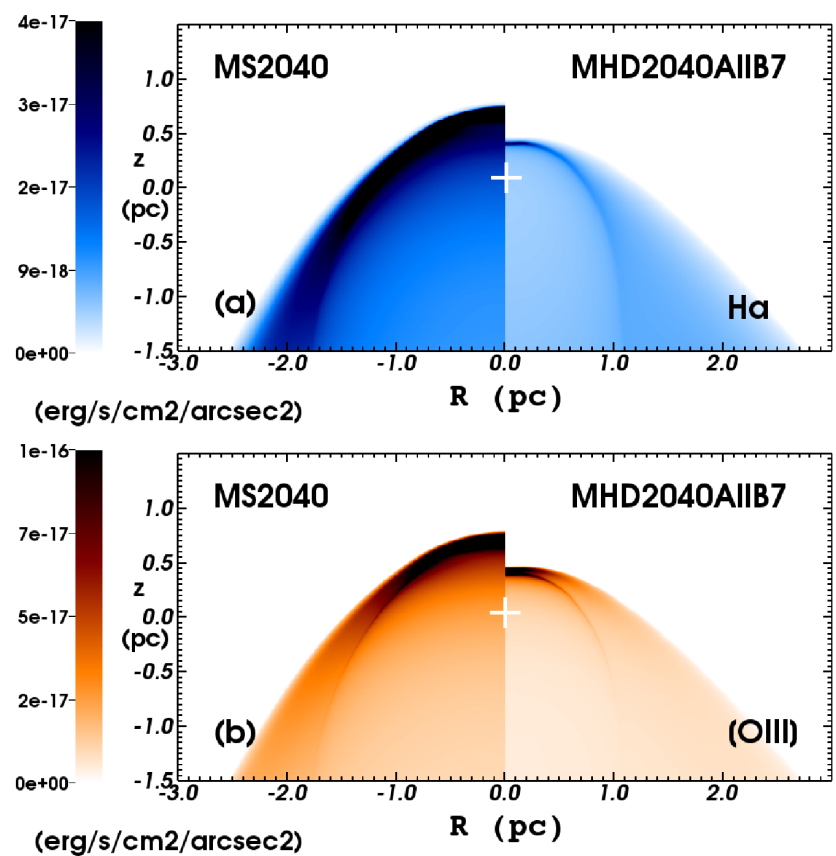

Figure 7. Surface brightness maps of $\mathrm{H} \alpha$ (a), [OIII] (b) surface brightness (in $\mathrm{erg} \mathrm{s}^{-1} \mathrm{~cm}^{-2} \operatorname{arcsec}^{-2}$ ), respectively, of our bow shock model generated by our initially $20 \mathrm{M}_{\odot}$ star moving with velocity $v_{\star}=40 \mathrm{~km} \mathrm{~s}^{-1}$. Quantities are calculated excluding the undisturbed ISM and plotted in the linear scale. The left-hand part of the panels refers to the hydrodynamical model MS2040, the right-hand part to the magneto-hydrodynamical model MHD2040AllB7. The crosses mark the position of the star. For the sake of comparison, these optical maps are presented as in Paper I.

and $60 \mu \mathrm{m}$ because they corresponds to the wavelengths at which stellar wind bow shocks are typically observed, see Sexton et al. (2015) and van Buren \& McCray (1988a); van Buren et al. (1995); Noriega-Crespo et al. (1997), respectively. Our SEDs and images are calibrated to such that we consider that the objects are located at a distance $1 \mathrm{pc}$ from the observer. 


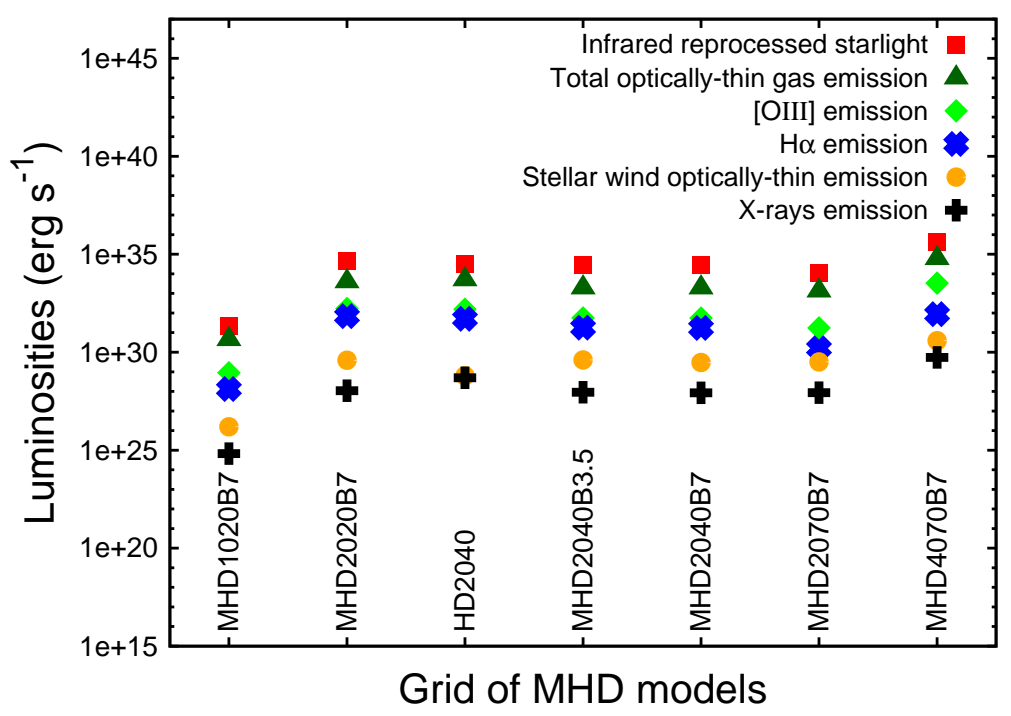

Figure 8. Bow shocks luminosities and feedback of our magneto-hydrodynamical models. We separate the infrared reprocessed starlight (red squares, in $\mathrm{erg} \mathrm{s}^{-1}$ ) and distinguish the total emission by optically-thin radiation from the bow shock (dark-green triangles, in erg s${ }^{-1}$ ) from the emission from the shocked wind material only (orange dots, in $\mathrm{erg} \mathrm{s}^{-1}$ ). Additionally, we show the luminosity from [OIII $\lambda 5007$ emission (pale green losanges, in erg s ${ }^{-1}$ ), the luminosity from $\mathrm{H} \alpha$ emission (blue crosses, in $\mathrm{erg} \mathrm{s}^{-1}$ ) and the X-rays luminosity in both the soft and hard energy bands $E>0.5 \mathrm{eV}\left(T>5.8 \times 10^{6} \mathrm{~K}\right)$. For the sake of comparison we add the feedback of the hydrodynamical model HD2040 corresponding to $B_{\mathrm{ISM}}=0 \mu \mathrm{G}$ (originally published in Paper I). The simulations labels are indicated under the corresponding values.

\subsection{Results: optically-thin emission}

In Table 4 we report the maximum surface brightness measured along the direction of motion of the stars in the synthetic emission maps build from our models at both the $\mathrm{H} \alpha$ and [OIII] $\lambda 5007$ spectral line emission. We find that the presence of an ISM magnetic field makes the $\mathrm{H} \alpha$ signatures fainter by about 1-2 orders of magnitudes whereas the [OIII] $\lambda 5007$ emission maps are about 1 order of magnitude fainter, respectively. The luminosity of stellar wind bow shocks is a volume integral (Paper I) and this volume decreases when a large ISM magnetic pressure compresses the nebula (Fig. 1 d,h). Thus, their surface brightness is fainter despite of the fact that the density and temperature of their shocked regions is similar (Fig. 12).

The ratio of our bow shocks models' maximum [OIII] and $\mathrm{H} \alpha$ maximum surface brightness increases in the presence of the magnetic field, e.g. the hydrodynamical model MS2040 has $\Sigma_{[\mathrm{OIII}]}^{\max } / \Sigma_{[\mathrm{H \alpha}]}^{\max } \approx 2.1$ whereas our model MHD2040AllB7 has $\Sigma_{[\mathrm{OIII}]]}^{\max } / \Sigma_{[\mathrm{H \alpha}]}^{\max } \approx 5.5$ if $B_{\mathrm{ISM}}=7 \mu \mathrm{G}$. We notice that the spectral line ratio $\Sigma_{[\mathrm{OIII}]]}^{\max } / \Sigma_{[\mathrm{H} \alpha]}^{\max }$ augments with the increasing space velocity of the star, e.g. our models MHD2020B7, MHD2040AllB7 and MHD2070B7 have $\Sigma_{[\mathrm{OIII}]]}^{\max } / \Sigma_{[\mathrm{H} \alpha]}^{\max } \approx 4.0,5.5$ and 25.0, respectively. This difference between [OIII] $\lambda 5007$ and $\mathrm{H} \alpha$ emission is more pronounced in our magneto-hydrodynamical simulations. As for our hydrodynamical study, the region of maximum emission peaks close to the contact discontinuity in the layer of shocked ISM material, in the region of the stagnation shock (Paper I, see also Figs.77,b).

The ISM magnetic field does not change the order relations we previously established with hydrodynamical bow shocks generated by main-sequence stars (Fig. 13a in Paper I), i.e. $L_{\text {wind }}<$ $L_{\mathrm{H} \alpha}<L_{\text {total }}<L_{\mathrm{IR}}$ (see orange dots, blue crosses of SaintAndrew, dark green triangles and black squares in Fig. 8 a, respectively). Additionally, as discussed above in the context of projected emission maps, we find that the optical spectral line emis- sion that we consider are such that $L_{\text {[OIII }]}>L_{\mathrm{H} \alpha}$. This confirms and extend to magneto-hydrodynamical bow shocks a result previously obtained by integrating the optically-thin emission in the range $8000 \leqslant T \leqslant 10^{6} \mathrm{~K}$ (Paper I). Our magneto-hydrodynamical bow shock models have $\mathrm{H} \alpha$ and [OIII] emission originating from the shocked ISM gas and their emission from the wind material is negligible ( $L_{\text {total }} / L_{\text {wind }} \approx 10^{-6}$ ). Moreover, we find that the bow shocks $\mathrm{X}$-rays emission are very small in all our simulations ( $L_{\mathrm{X}} / L_{\text {wind }} \approx 10^{-1}$, see black crosses in Fig. 8 ).

\subsection{Results: dust continuum infrared emission}

\subsubsection{Spectral energy distribution}

Fig. 9p plots a comparison betwenn the SEDs of two bow shock models generated by our $20 \mathrm{M}_{\odot}$ star moving with velocity $40 \mathrm{~km} \mathrm{~s}^{-1}$, either through an unmagnetized ISM (model MS2040, solid blue line) or in a medium with $B_{\mathrm{ISM}}=7 \mu \mathrm{G}$ (model MHD2040AllB7, dotted red line) for a viewing angle of the nebulae of $\phi=0^{\circ}$. The figure represents the flux density $F_{\lambda}$ (in Jy) as a function of the wavelength $\lambda$ (in $\mu \mathrm{m}$ ) for the waveband including the $0.01 \leqslant \lambda \leqslant 2000 \mu \mathrm{m}$. The star is responsible for the component in the range $0.01 \leqslant \lambda \leqslant 10 \mu \mathrm{m}$ that corresponds to a black body spectrum of temperature $T_{\text {eff }}=33900 \mathrm{~K}$ (see Table 1) while the circumstellar dust produces the feature in the waveband $10 \leqslant \lambda \leqslant 2000 \mu \mathrm{m}$. The bow shock's component is in the waveband including the wavelengths at which stellar wind bow shock are typically recorded, e.g. at $60 \mu \mathrm{m}$ van Buren \& McCray (1988a); van Buren et al. (1995); Noriega-Crespo et al. (1997).

The SED of the magnetized bow shock has a slightly larger flux than the SED of the hydrodynamical bow shock in the waveband $10 \leqslant \lambda \leqslant 30 \mu \mathrm{m}$, because its smaller size makes the shell of dense ISM gas closer to the star, increasing therefore the dust temperature (Fig. 9 b). At $\lambda \approx 30 \mu \mathrm{m}$, the hydrodynamical bow shock emits by slightly more than half an order of magni- 

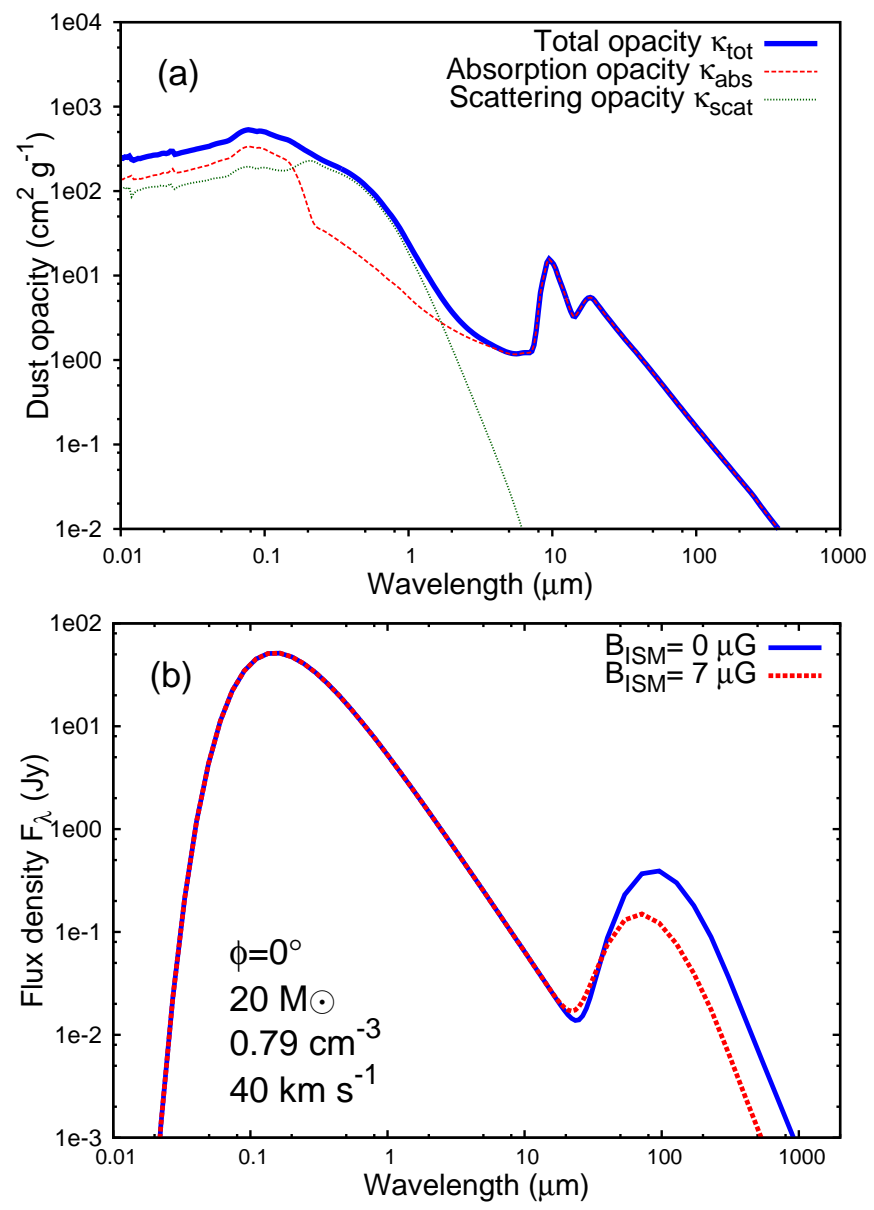

Figure 9. Top panel: dust opacities used in this study, inspired from Acreman et al. 2016). The figure shows the total opacity $\kappa_{\text {tot }}$ (blue solid thick line), the absorption opacity $\kappa_{\text {abs }}$ (red dotted thin line) and the scattering opacity $\kappa_{\text {scat }}$ (green dashed thin line). Bottom panel: spectral energy distributions of our model involving a $20 \mathrm{M}_{\odot}$ star moving with a velocity of $40 \mathrm{~km} \mathrm{~s}^{-1}$, considered in the hydrodynamical (model MS2040 with $B_{\mathrm{ISM}}=0 \mu \mathrm{G}$, solid blue line) and in the magneto-hydrodynamical contexts (model MHD2040AllB7 with $B_{\mathrm{ISM}}=7 \mu \mathrm{G}$, dotted red line). The plot shows the flux density $F_{\lambda}$ (in Jy) as a function of the wavelength $\lambda$ (in $\mu \mathrm{m}$ ) for an inclination angle $\phi=0^{\circ}$ of the bow shock.

tude than the magnetized nebula, e.g. at $\lambda \approx 60 \mu \mathrm{m}$ our model MS2040 has a density flux $F_{\lambda} \approx 3 \times 10^{-1}$ Jy whereas our model MHD2040AllB7 shows $F_{\lambda} \approx 1 \times 10^{-1} \mathrm{Jy}$, respectively. This is consistent with the previously discussed reduction of the projected optical emission of our bow shocks. This relates to the changes in size of the nebulae induced by the inclusion of the magnetic field in our simulations, which reduces the mass of dust in the structure responsible for the reprocessing of the starlight, e.g. our models MS2040 and MHD2040AllB7 contain about $M_{\mathrm{d}} \approx 3 \times 10^{-2} \mathrm{M}_{\odot}$ and $M_{\mathrm{d}} \approx 2 \times 10^{-3} \mathrm{M}_{\odot}$, respectively, where $M_{\mathrm{d}}$ is the dust mass trapped into the nebulae. The reduced mass of dust into the magnetized bow shock absorbs a lesser amount of the stellar radiation and therefore re-emits a smaller quantity of energy, reducing $F_{\lambda}$ in the waveband $\lambda \geqslant 30 \mu \mathrm{m}$ (Fig. $9 \mathrm{~b}$ ). Note that the infrared surface brightness of a bow shock is also sensible to the density of its ambient medium, i.e. $F_{\lambda}$ is much larger in the situation of a runaway star moving in a medium with $n_{\mathrm{ISM}} \simeq 1000 \mathrm{~cm}^{-3}$ Acreman et al. 2016).

\subsubsection{Synthetic infrared emission maps}

Our Fig. 10 plots a series of synthetic infrared emission maps of our bow shock models produced by an initially $20 \mathrm{M}_{\odot}$ star moving with velocity $40 \mathrm{~km} \mathrm{~s}^{-1}$ in its purely hydrodynamical (MS2040) or magneto-hydrodynamical configuration (MHD2040AllB7) at the wavelengths corresponding to the central wavelengths of the IRAS facility's main broadband images (van Buren \& McCray 1988b), i.e. $\lambda=25 \mu \mathrm{m}$ (left column of panels), $60 \mu \mathrm{m}$ (middle column of panels) and $100 \mu \mathrm{m}$ (right column of panels). The maps are represented with an inclination angle of $\phi=30^{\circ}$ (Fig. 10 , e,i), 45 (Fig. 10 b,f,j), $60^{\circ}$ (Fig.10, g,k) and $90^{\circ}$ (Fig. 10 d,h,l) with respect to the plane of the sky and the projected flux is plotted in units of $\mathrm{erg} \mathrm{s}^{-1} \mathrm{~cm}^{-2} \operatorname{arcsec}^{-2}$. As in the context of their optical emission (Fig. 7), the overall size of the infrared magnetized bow shocks is smaller than in the hydrodynamical case because of the reduction of their stand-off distance $R(0)$, see, e.g. Fig. 10, e,j. The global morphology of our infrared bow shock nebulae does not change significantly. It remains a single, bright arc at the front of an ovoid structure that is symmetric with respect to the direction of motion of the runaway star and extended to the trail $(z \leqslant 0)$ of the bow shocks due to the supersonic motion of the star Acreman et al. (2016). In the hydrodynamical case, the region of maximum emission is the region containing the ISM dust which temperature is smaller than a few $10^{4} \mathrm{~K}$, i.e. between the contact discontinuity and the forward shock of the bow shock (Paper I, Acreman et al. 2016) whereas in the magnetized case, the maximum emission is reduced to a thin region close to the discontinuity between hot stellar wind and colder ISM. Both the shocked stellar wind and the shocked ISM of the bow shock do not contributes to these emission because the material is too hot.

Fig. 11 reports cross-sections taken along the direction of motion of the bow shock and comparing their surface brightesses at several wavebands $\lambda$ and viewing angles $\phi$. It illustrates that, as in the case of the optical emission, the presence of the ISM magnetic field makes the bow shocks slightly dimmer, e.g. for $\phi=45^{\circ}$ our model has a maximal surface brighness of $\Sigma_{100 \mu \mathrm{m}}^{\max } \approx$ $4.3 \times 10^{-17} \mathrm{erg} \mathrm{s}^{-1} \mathrm{~cm}^{-2} \operatorname{arcsec}^{-2}$ whereas $\Sigma_{100 \mu \mathrm{m}}^{\max } \approx 2.6 \times$ $10^{-17} \mathrm{erg} \mathrm{s}^{-1} \mathrm{~cm}^{-2} \operatorname{arcsec}^{-2}$ for $B_{\mathrm{ISM}}=0$ and $7 \mu \mathrm{G}$, respectively. Fig. 12 shows different cross-sections of the projected infrared emission the magnetized bow shock of our initially $20 \mathrm{M}_{\odot}$ star moving with velocity $v_{\star}=40 \mathrm{~km} \mathrm{~s}^{-1}$. The emission at $\lambda=$ $60 \mu \mathrm{m}$ is more important that at $\lambda=25 \mu \mathrm{m}$ and at $\lambda=100 \mu \mathrm{m}$, e.g. it peaks at $\Sigma_{60 \mu \mathrm{m}}^{\max } \approx 8.2 \times 10^{-17} \mathrm{erg} \mathrm{s}^{-1} \mathrm{~cm}^{-2} \operatorname{arcsec}^{-2}$ whereas $\Sigma_{25 \mu \mathrm{m}}^{\max } \approx 2.6 \times 10^{-17} \mathrm{erg} \mathrm{s}^{-1} \mathrm{~cm}^{-2} \operatorname{arcsec}^{-2}$ and $\Sigma_{100 \mu \mathrm{m}}^{\max } \approx 3.0 \times 10^{-17} \mathrm{erg} \mathrm{s}^{-1} \mathrm{~cm}^{-2} \operatorname{arcsec}^{-2}$, respectively, at a distance of $0.55 \mathrm{pc}$ from the star and assuming an inclination angle of the bow shock of $\phi=45^{\circ}$ (Fig. 12 all ). All our models have similar behaviour of their infrared surface brightness as a function of $\lambda$ and $\phi$. Note also that the evolution of the position of the stand-off distance of the bow shock is consistent with the study of Acreman et al. (2016) in the sense that it increases at larger $\phi$ (Fig. 12b).

\subsection{Implications of our results and discussion}

\subsubsection{Bow shocks Ho and [OIII] observability}

The surface brightnesses at $\mathrm{H} \alpha$ and [OIII] $\lambda 5007$ spectral line emission of our stellar wind bow shocks reported in Table 4.4.4 indicate that (i) the presence of the ISM magnetic field makes their projected emission $\Sigma_{\mathrm{H} \alpha}$ and $\Sigma_{[\mathrm{OIII}}$ fainter by two and 1-2 orders of magnitude and (ii) that this reduction of the nebulae's 

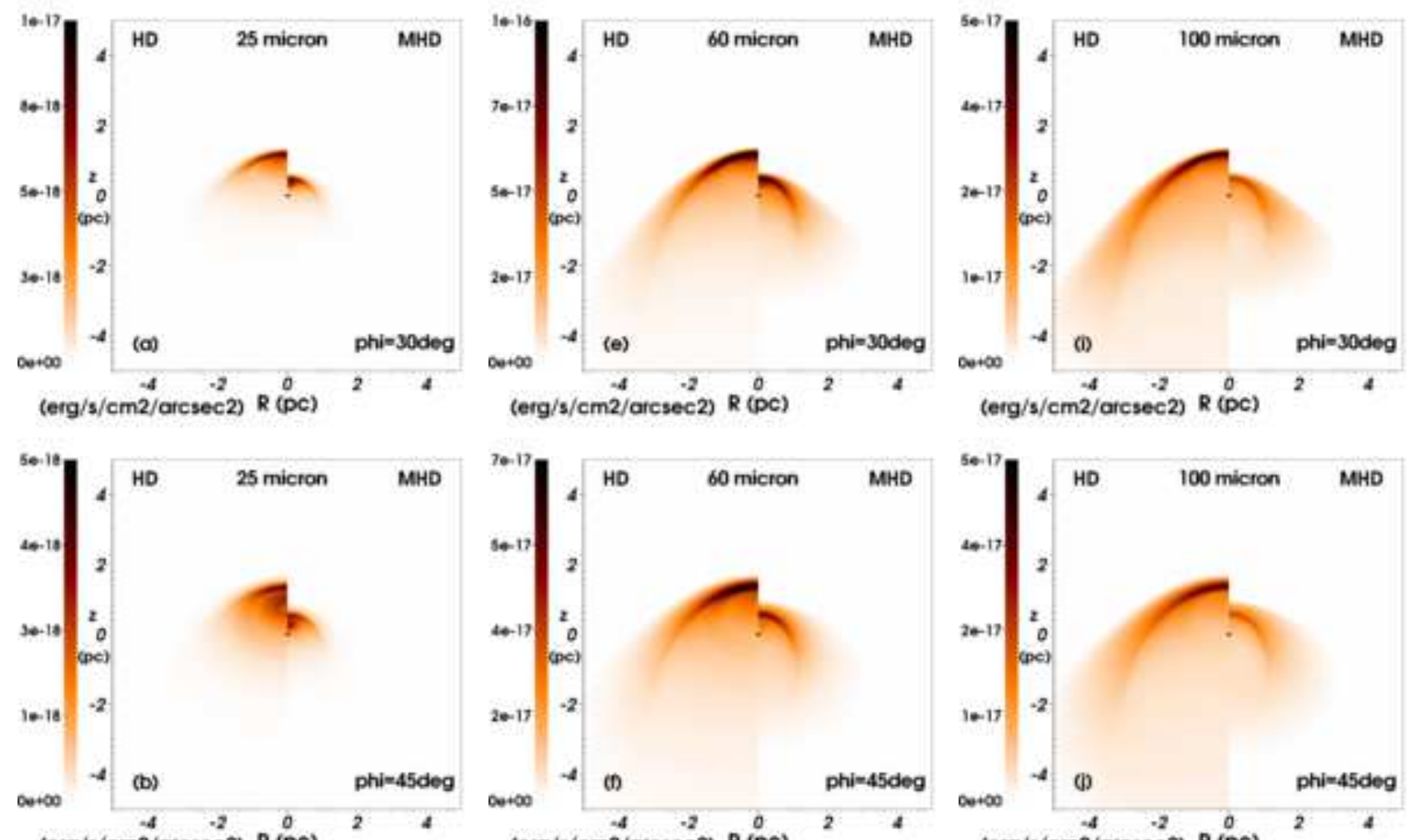

(erg/s/cm2/arcsec2) R (pc)
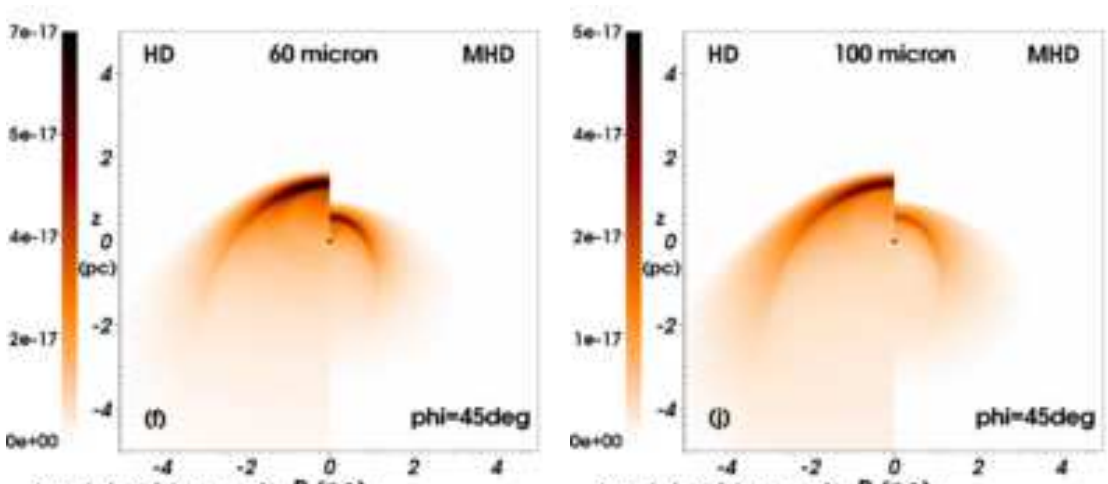

(erg/s/cm2/arcsec2) R (pc)

(erg/s/cm2/arcsec2) R (pC)
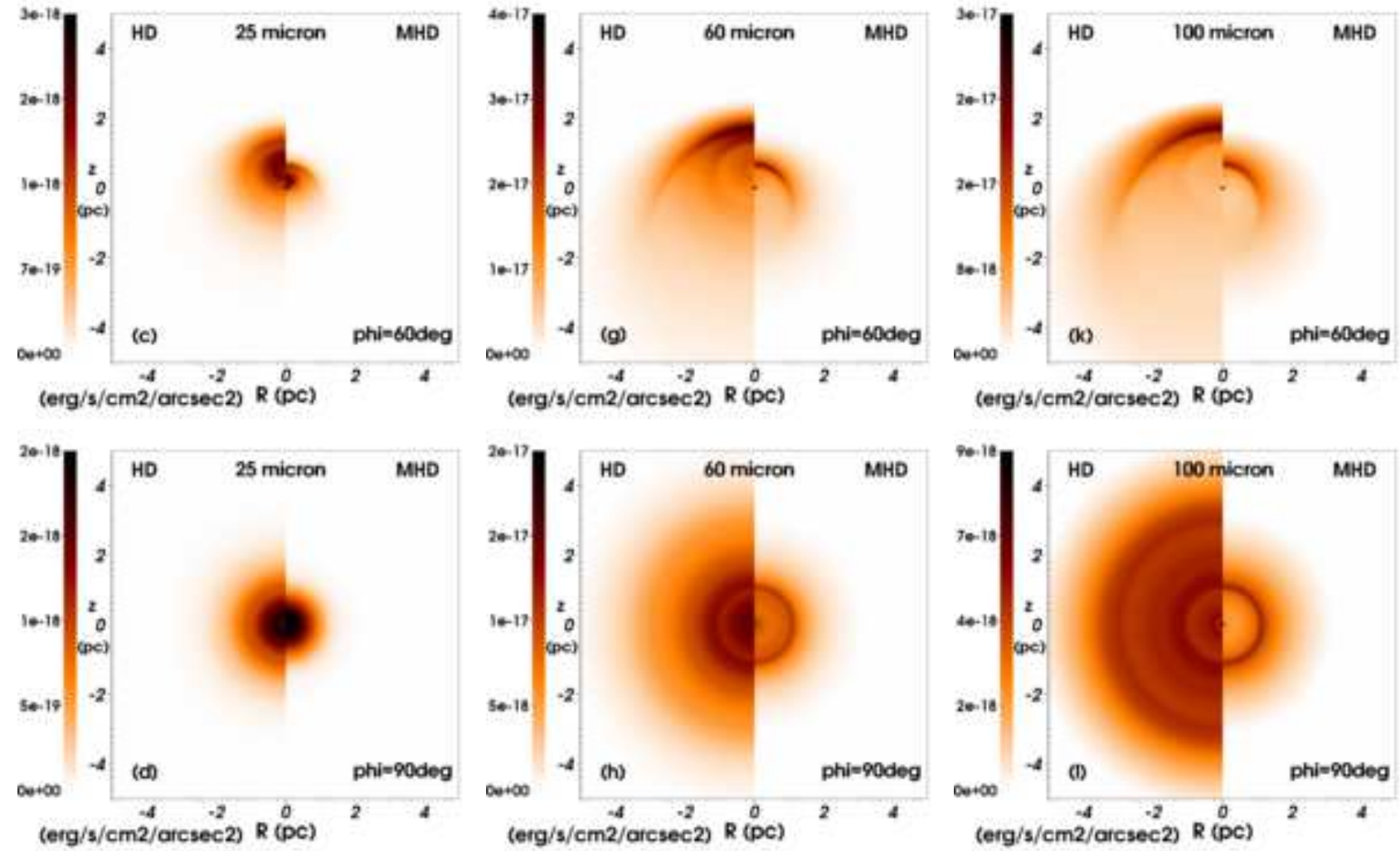

Figure 10. Isophotal infrared emission maps of our bow shock models MS2040 and MHD2040AllB7. It represents our initially $20 \mathrm{M} \odot$ star moving with velocity $40 \mathrm{~km} \mathrm{~s}^{-1}$ as seen at wavelengths $\lambda=25$ (a-d), $60 \mu \mathrm{m}(\mathrm{e}-\mathrm{h})$ and $100 \mu \mathrm{m}$ (i-1). The projected flux is in units of $\mathrm{erg} \mathrm{s}^{-1} \mathrm{~cm}^{-2}$ arcsec ${ }^{-2}$. The maps are generated with an inclination angle of $\phi=30(\mathrm{a}, \mathrm{e}, \mathrm{i}), 45(\mathrm{~b}, \mathrm{f}, \mathrm{j}), 60(\mathrm{c}, \mathrm{g}, \mathrm{k})$ and $90^{\circ}(\mathrm{d}, \mathrm{h}, \mathrm{l})$ with respect to the plane of the sky. For each panel, the surface brightness is plotted in the linear scale and its maximum corresponds to the maximum of the hydrodynamical (left) and magneto-hydrodynamical bow shock models (right). 


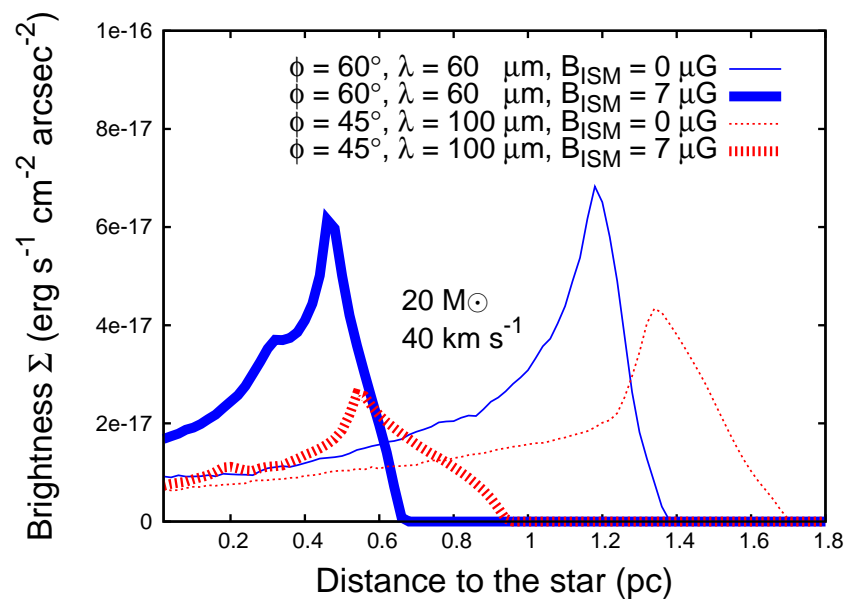

Figure 11. Cross-sections taken through the direction of motion of the bow shock of our initially $20 \mathrm{M}_{\odot}$ star moving in a medium with velocity $v_{\star}=$ $40 \mathrm{~km} \mathrm{~s}^{-1}$, both in a medium with $B_{\mathrm{ISM}}=0$ and $7 \mu \mathrm{G}$. The emission are shown for a viewing angle of $\phi=45^{\circ}$ and at the waveband $\lambda=$ $60 \mu \mathrm{m}$ (dotted red curves) and for $\phi=60^{\circ}$ at $\lambda=100 \mu \mathrm{m}$ (solid blue curves). The surface brightness (in erg s${ }^{-1} \mathrm{~cm}^{-2} \operatorname{arcsec}^{-2}$ ) is plotted as a function of the distance to the star (in pc). The position of the star is located at the origin.

emission is more important as the strength of the B field is larger. Consequently, the emission signature of a purely hydrodynamical bow shock model that is above the the diffuse emission sensitivity threshold of, e.g. the SuperCOSMOS H-Alpha Survey (SHS) of $\Sigma_{\text {SHS }} \approx 1.1-2.8 \times 10^{-17} \mathrm{erg} \mathrm{s}^{-1} \mathrm{~cm}^{-2} \operatorname{arcsec}^{-2}$ can drop down below it once the ISM magnetic field is switched-on. As an example, our hydrodynamical model of a $20 M_{\odot}$ star moving with velocity $v_{\star}=70 \mathrm{~km} \mathrm{~s}^{-1}$ (Paper I) could be observed since it has $\Sigma_{\mathrm{H} \alpha} \approx$ $1.5 \times 10^{-16} \geqslant \Sigma_{\text {SHS }}$ whereas our magneto-hydrodynamical model of the same runaway star has $\Sigma_{\mathrm{H} \alpha} \approx 8.0 \times 10^{-18} \ll \Sigma_{\text {SHS }}$ and would be invisible regarding to the SHS facility (our Table 4).

This may explain why not so many stellar wind bow shocks are discovered at $\mathrm{H} \alpha$ around isolated, hot massive stars, despite of the fact the ionisation of their circumstellar medium must produce such emission (Brown \& Bomans 2005). Since $\Sigma_{\mathrm{H} \alpha} \propto n^{2}$ (see Appendix A of Paper I), it implies that the more diluted the ISM constituting the surrounding of an exiled star, i.e. the higher the runaway star's Galactic latitude, the smaller the probability to observe its bow shock at $\mathrm{H} \alpha$. In other words, the search for bow shocks at this wavelength may work well within the Galactic plane or in relatively dense regions of the ISM. Note also that in the presence of the magnetic field, all models have $\Sigma_{[\mathrm{OIII}]} / \Sigma_{\mathrm{H \alpha}}>1$, which is consistent with the discovery of the first bow-shock-producing massive stars $\zeta$ Ophiuchi in [OIII] $\lambda 5007$ emission.

\subsubsection{Surrounding $H \mathrm{II}$ region and dust composition}

Massive stars release huge amount of ultraviolet photons (Diaz-Miller et al. 1998) that ionize the hydrogen constituting their surroundings (Dyson 1975), giving birth to an H II region overwhelming the stellar wind bubble of the star (Weaver et al. 1977; van Marle 2006). In the case of a runaway star, the stellar motion produces a bow shock surrounded by a cometary H II region (Raga 1986; Mac Low et al. 1991; Raga et al. 1997; Arthur \& Hoare 2006; Zhu et al. 2015), which presence in our study is simply taken into account assuming that the ambient medium of the star is fully ionized, however, we neglect its turbulent internal structure. The gas that is between the forward shock of the bow shock and the outer part of the HII region is filled by ISM dust that emits infrared thermal emission by efficiently reprocessing the stellar radiation, i.e. it is brighter that the emission by gas cooling (Paper I).

While our study shows that our nebulae are brighter at $60 \mu \mathrm{m}$ (Fig. 12), i.e. at the waveband at which catalogues of bow shocks from OB stars have been compiled (van Buren \& McCray 1988a; van Buren et al. 1995; Noriega-Crespo et al. 1997), the study of Mackey et al. (2016) compared the respective brightnesses of the front of a distorted circumstellar bubble with the outer edge of its surrounding H II region and find the $24 \mu \mathrm{m}$ waveband to be ideal to observe the structure generated by the stellar wind. However, the presence of the ISM background magnetic field makes our infrared arc smaller and slightly dimmer, i.e. more difficult to detect in the case of a distant runaway star which could explain why a large proportion of observed H II regions do not contain dust-free cavities encircled with bright mid-infrared arcs (Sharpless 1959; Churchwell et al. 2006; Wachter et al. 2010; Simpson et al. 2012). Further radiation magneto-hydrodynamics simulations are required to fully assess the question of the infrared screening of stellar wind bow shocks by their own H II regions, particularly for an ambient medium corresponding to the Galactic plane $\left(n_{\mathrm{ISM}} \simeq 1 \mathrm{~cm}^{-3}\right)$.

Following Pavlyuchenkov et al. (2013), we consider that the dust filling the HII region and penetrating into the bow shock is similar of that of the ISM. Our radiative transfer calculations nevertheless suffer from uncertainties regarding to the composition of this ISM dust. Our mixture is made of Silicates (Draine \& Lee 1984) which could be modified, e.g. changing the slope of the dust size distribution. Particularly, the inclusion of very small grains such as polycyclic aromatic hydrocarbon (PAHs, see Wood et al. 2008) may be an appropriate update of the dust mixture, as it have been shown to be necessary to fit observations of mid-infrared bow shocks around $\mathrm{O}$ stars in dense medium in M17 and RCW 49 (Povich et al. 2008). Enlarging our work in a wider study, e.g. scanning the parameter space of the quantities governing the formation of Galactic stellar wind bow shocks $\left(v_{\star}, n_{\mathrm{ISM}}, M_{\star}\right)$ in order to discuss both their SEDs and infrared images will be considered in a follow-up paper, e.g. performing a systematic post-processing of the grid of bow shock simulations of Meyer et al. (2016) with RADMC-3D. Then, thorough comparison of numerical simulations with, e.g. the IRAS observations of van Buren \& McCray (1988a); van Buren et al. (1995); Noriega-Crespo et al. (1997) would be achievable.

\subsubsection{Shaping of the circumstellar medium of runaway massive stars at the pre-supernova phase}

It has been shown in the context of Galactic, high-mass runaway stars, that the pre-shaped circumstellar medium in which these stars die and explode as a type II supernova is principally constituted of its own main-sequence wind bubble, distorted by the stellar motion. Further evolutionary phase(s) produce additional bubble(s) and/or shell(s) whose evolution is contained inside the initial bow shock (Brighenti \& D'Ercole 1994, 1995a). The expansion of the subsequent supernova shock wave is strongly impacted by the progenitor's pre-shaped circumstellar medium inside which it develops initially (see, e.g. Cox et al. 1991). Particularly, the more welldefined and stable the walls of the tunnel formed by the reverse shock of the bow shock are, the easier the channeling the supernovae ejecta inside it (see in particular Appendix A of Meyer et al. 2015, and references therein). 

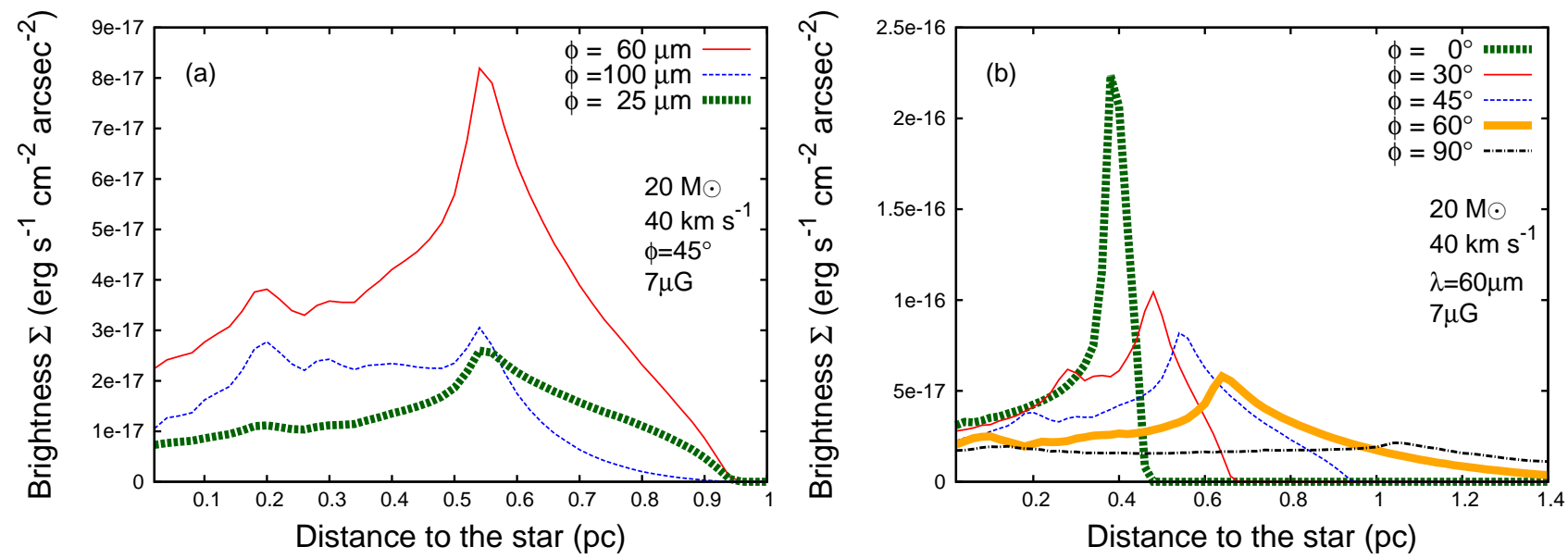

Figure 12. Cross-sections taken through the direction of motion of the bow shock of our initially $20 \mathrm{M}_{\odot}$ star moving in a medium with $B_{\mathrm{ISM}}=7 \mu \mathrm{G}$ with velocity $v_{\star}=40 \mathrm{~km} \mathrm{~s}^{-1}$. The emission are shown for the principal broadband images of the IRAS telescope for a viewing angle $\phi=45^{\circ}$ (a) and for the wavelength $\lambda=60 \mu \mathrm{m}$ as a function of different viewing angle $\phi(\mathrm{b})$. The surface brightness (in $\mathrm{erg} \mathrm{s}^{-1} \mathrm{~cm}^{-2} \operatorname{arcsec}^{-2}$ ) is plotted as a function of the distance to the star (in pc). The position of the star is located at the origin.

Our study shows that the presence of background ISM magnetic field aligned with the direction of motion of a main-sequence runaway star inhibits the growth of both shear instabilities that typically affect these circumstellar structures (Fig. 1). Consequently, a planar-aligned magnetic field would further shape the reverse shock of moving stars' bow shocks as a smooth tube in which shocks waves could be channeled as a jet-like extension, e.g. as in Cox et al. (1991). Additionally, the shock wave outflowing out of the forward shock of circumstellar structures of runaway stars that are sufficiently dense to make their subsequent supernova remnant asymmetric (Meyer et al. 2015) would be more collimated along the direction of motion of its progenitor and/or ambient magnetic field. This may produce additional asymmetries to the elongated shape of supernovae remnants exploding in a magnetized ISM (Rozyczka \& Tenorio-Tagle 1995).

\subsubsection{The case of the hot runaway star $\zeta$ Ophiuchi}

The O9.5 V star $\zeta$ Ophiuchi is the Earth's closest massive, main-sequence runaway star. Infrared observations, e.g. with the WISE $3.4 \mu \mathrm{m}$ facility (band W1, Wright et al. 2010, see Fig. 13. highlighted the complex topology of its stellar wind bow shock, originally discovered in [OIII] $\lambda 5007$ spectral line (Gull \& Sofia 1979) and further observed in the infrared waveband (van Buren \& McCray 1988b). The properties of the particular, non-axisymmetric shape of its circumstellar nebula which moves in the HII region Sh 2-27 (Sharpless 1959 ) is studied in a relatively large literature (see Mackey et al. 2013, and references therein). The mass-loss of $\zeta$ Ophiuchi has been estimated in the range $\dot{M}_{\zeta} \approx 1.58 \times 10^{-9}-1.43 \times$ $10^{-7} \mathrm{M}_{\odot} \mathrm{yr}^{-1}$ Gvaramadze et al. 2012), which, according to Eq. 21 , taking $R(0) \approx 0.16$ pc (Gvaramadze et al. 2012), adopting $v_{\star} \approx 26.5 \mathrm{~km} \mathrm{~s}^{-1}$ and considering a typical OB star wind velocity of $v_{\mathrm{w}} \approx 1500 \mathrm{~km} \mathrm{~s}^{-1}$, constrains its ambient medium density to $n_{\zeta} \approx 3-4 \mathrm{~cm}^{-3}$ (cf. Gull \& Sofia 1979).

Assuming (i) the magnetisation of the close surrounding of $\zeta$ Ophiuchi to be $B_{\mathrm{ISM}}=7 \mu \mathrm{G}$ (Mackey et al. 2013), (ii) that the

${ }^{4}$ http://wise2.ipac.caltech.edu/docs/release/allsky/ conditions for switch-on shocks to be permitted are fulfilled, i.e. plasma and Alfvénic velocities are normal to the shock, and (iii) considering that its ISM properties are, in addition to the above presented quantities, such that $\gamma=1.67, T_{\text {ISM }}=8000 \mathrm{~K}$, it comes that $\beta \geqslant 2 / \gamma$ and $M_{\mathrm{A}}<1$. This indictes that, under our hypothesises, the ambient medium of $\zeta$ Ophiuchi does not allow the existence of switch-on shocks. Consequently, the imperfect shape of its bow shock (Fig. 13) may not be explained invoking the particular double-front topology of bow shocks that can be produced in such regime, but rather by the presence of a background ISM magnetic field whose direction is not aligned with respect to the motion of the star. Further tri-dimensional magneto-hydrodynamical models are needed in order to assess the question of $\zeta$ Ophiuchi's background ISM magnetic field direction, the position of its contact discontinuity and a more precise estimate of its stellar wind mass-loss.

\subsubsection{The case of runaway cool stars}

Our results apply to bow shocks generated by hot, main-sequence OB stars that move through the hot ionized gas of their own H II region (Raga et al. 1997) and archetype of which is the nebulae surrounding $\zeta$ Ophiuchi (see above discussion). Externallyphotoionized cool runaway stars that move rapidly in the H II region produced by an other source of ionizing radiation have particularly bright optical emission, see e.g. the cases of the red supergiant Betelgeuse (Mohamed et al. 2012; Mackey et al. 2014) and IRC-10414 (Mever et al. 2014). These circumstellar structures are themselves sensitive to the presence of even a weak ISM background magnetic field of a few $\mu \mathrm{G}$ (van Marle et al. 2014). Consequently, one can expect that the inclusion of such a field in numerical models tailored to these objects would affect their associated synthetic emission maps and update the current estimate of their driving star's mass loss and/or ambient medium density (Mever et al. 2016).

According to the fact that the warm phase of the ISM is typically magnetized, the reduction of both optical and infrared surface brightnesses of circumstellar structures generated by massive stars should be a rather common phenomenon. In particular, it should also concern bow shocks of OB runaway stars once they have evolved through the red supergiant phase ( $\mathrm{Pa}-$ 
per I). However, the proportion of red supergiant stars amongst the population of all runaway massive stars should be similar to the proportion of red supergiant with respect to the population of static OB stars, which is, to the best of our knowledge, contradicted by observations. The recent study of van Marle et al. (2014) shows that a background ISM magnetic field can inhibits the growth of shear instabilities, i.e. forbids the development of potentially bright infrared knots, in the bow shock of Betelgeuse, and, this may participate in explaining why the scientific literature only reports 4 known runaway red superigant stars, amongst which only 3 have a detected bow shock, i.e. Betelgeuse (Noriega-Crespo et al. 1997), IRC-10414 (Meyer et al. 2014) and $\mu$ Cep (Cox et al. 2012). The extragalactic, hyperveloce red supergiant star J004330.06+405258.4 in M31 has all kinematic characteristics to generate a bow shock but it has not been observed so far (Evans \& Massey 2015). This remark is also valid for bow shocks generated by runaway massive stars experiencing other evolutionary stages such as the so-called blue supergiant phase (see, e.g. Kaper et al. 1997).

\subsubsection{Comparison with the bow shock around the Sun}

The Sun is moving into the warm phase of the ISM (McComas et al. 2015) and the properties of its ambient surrounding, the so-called local interstellar medium (LISM) are similar to the ISM in which our runaway stars move, especially in terms of Alfvénic Mach number and plasma $\beta$ (Florinski et al. 2004; Burlaga et al. 2015). The study of the interaction between our Sun and the LISM led to a large literature, including, amongst other, numerical investigations of the bow shock formed by the solar wind (see, e.g. Pogorelov \& Matsuda 1998; Baranov \& Malama 1993; Zank 2015, and references therein). If obvious similitudes between the bow shock of the Sun and those of our massive stars indicate that the physical processes governing the formation of circumstellar nebulae around OB stars such as electronic thermal conduction or the influence of the background local magnetic field have to be included in the modelling of those structures (Zank et al. 2009), nevertheless, the bow shock of the Sun is, partly due to the differences in terms of effective temperature and wind velocity, on a totally different scale. Further resemblances with bow-like nebulae from massive stars are therefore mostly morphological.

As a low-mass star $\left(<8 \mathrm{M}_{\odot}\right)$, the Sun is much cooler $\left(T_{\text {eff }} \approx\right.$ $6000 \mathrm{~K})$ than the runaway OB stars considered in the present work $\left(T_{\text {eff }}>20000 \mathrm{~K}\right)$ and its mass-loss $\left(\dot{M}_{\odot} \approx 10^{-14} \mathrm{M}_{\odot} \mathrm{yr}^{-1}\right)$ is much smaller than that of a main-sequence star with $M_{\star} \geqslant 20 \mathrm{M}_{\odot}$ (our Table 1), which makes its stellar luminosity fainter by several orders of magnitude $\left(L_{\star} / L_{\odot} \geqslant 10^{3}\right)$. Moreover, the solar wind velocity at $1 \mathrm{AU}$ is about $350 \mathrm{~km} \mathrm{~s}^{-1}$ (Golub \& Pasachoff 1997) whereas our OB stars have larger wind velocities $\left(>1000 \mathrm{~km} \mathrm{~s}^{-1}\right.$, see Table 11. Stellar winds from solar-like stars consequently develop a smaller ram pressure and expel less linear momentum than massive stars such as our $20 \mathrm{M}_{\odot}$ star and their associated corresponding circumstellar structures, i.e. wind bubbles or bow shocks are scaled down to a few tens or hundreds of AU. Note also that the Sun is too cool to produce ionizing radiations and generated an $\mathrm{H}$ II region that is susceptible screen its optical/infrared wind bubble. In other words, if the numerical methods developed to study the bow shock surrounding the Sun are similar to the ones utilised in our study, the solar solutions are more appropriated to investigate the surroundings of cool, low-mass stars such as, asymptotic giant stars (AGB), see (Wareing et al. 2007b. a; Raga et al. 2008; Esquivel et al. 2010; Villaver et al. 2012; Chiotellis et al. 2016), or the trails let by planetesimals moving in stellar systems presenting a common envelope (see Thun et al. 2016, and references therein).

Early two-dimensional numerical models of the solar neighbourhood were carried out assuming that the respective directions of both the Sun's motion and the LISM magnetic field are considered as parallel, as we hereby do with our massive stars (Pogorelov \& Matsuda 1998). More sophisticated simulations have produced three-dimensional models in which the Sun moves obliquely through the LISM (see, e.g. Baranov et al. 1996; Boley et al. 2013). Such investigation is observationally motivated by the perturbated and non-uniform appearance of the heliopause, e.g. the boundary between the interplanetary and interstellar medium (Kawamura et al. 2010) which revealed the need for $3 \mathrm{D}$ calculations, able to report the non-stationary character of the trail of the bow shock of the Sun (Washimi \& Tanaka 1996; Linde et al. 1998; Ratkiewicz et al. 1998). Those models are more complex than our simplistic two-dimensional simulations and investigate, e.g. the charges exchanges arising between the stellar wind and the LISM (Fitzenreiter et al. 1990). These studies also highlighted the complexity and fragility of such models, e.g. regarding to the variety of instable MHD discontinuities that affects shock waves propagating through a magnetized flow and differentiating the shocks from purely hydrodynamical discontinuities described by the Rankine-Hugoniot (see also de Sterck et al. 1998; de Sterck \& Poedts 1999). Additionally, those solutions are affected by the spatial resolution of the calculations and the inclusion of numerical viscosity in the models (Lopez et al. 2011; Wang et al. 2014, and references therein).

Finally, let mention an other obvious difference between bow shock of the Sun and the nebulae generated by the runaway OB stars that we model. The proximity of the Earth with the Sun makes it easier to be studied and analysed by means of, e.g. radio observations (Baranov et al. 1975) while its innermost substructures are directly reachable with spacecrafts such as Voyager 1 and Voyager 25 . Their missions partly consisted in leaving the neighbourhood of our Sun in order to explore the heliosheath, i.e. the layer corresponding to the region of shocked solar wind that is between the contact discontinuity (the heliopause) and the reverse shock of the solar bow shock (the wind termination shock). The Voyager engines crossed the outermost edge of the solar system between 2004 and 2007 at a the expected distance of 94 and 84 AU from the Earth (Linde et al. 1998), giving the first experimental data on the physics of the interstellar medium (Chalov et al. 2016). Those measures proved the existence of the solar bow shock, but also highlighted the particular conditions of the outer space in terms of magnetic phenomenon (Richardson 2016) and effects of cosmic rays (Webber 2016). In order to make our models more realistic, those physical processes should be taken into account into future simulations of bow shocks from runaway high-mass stars.

\section{CONCLUSION}

In this study, we presented magneto-hydrodynamical models of the circumstellar medium of runaway, main-sequence, massive stars moving supersonically through the plane of the Galaxy. Our two-dimensional simulations first investigated the conjugated effects of optically-thin radiative cooling and heating together

\footnotetext{
${ }^{5}$ http://voyager.jpl.nasa.gov/mission/interstellar.html
} 


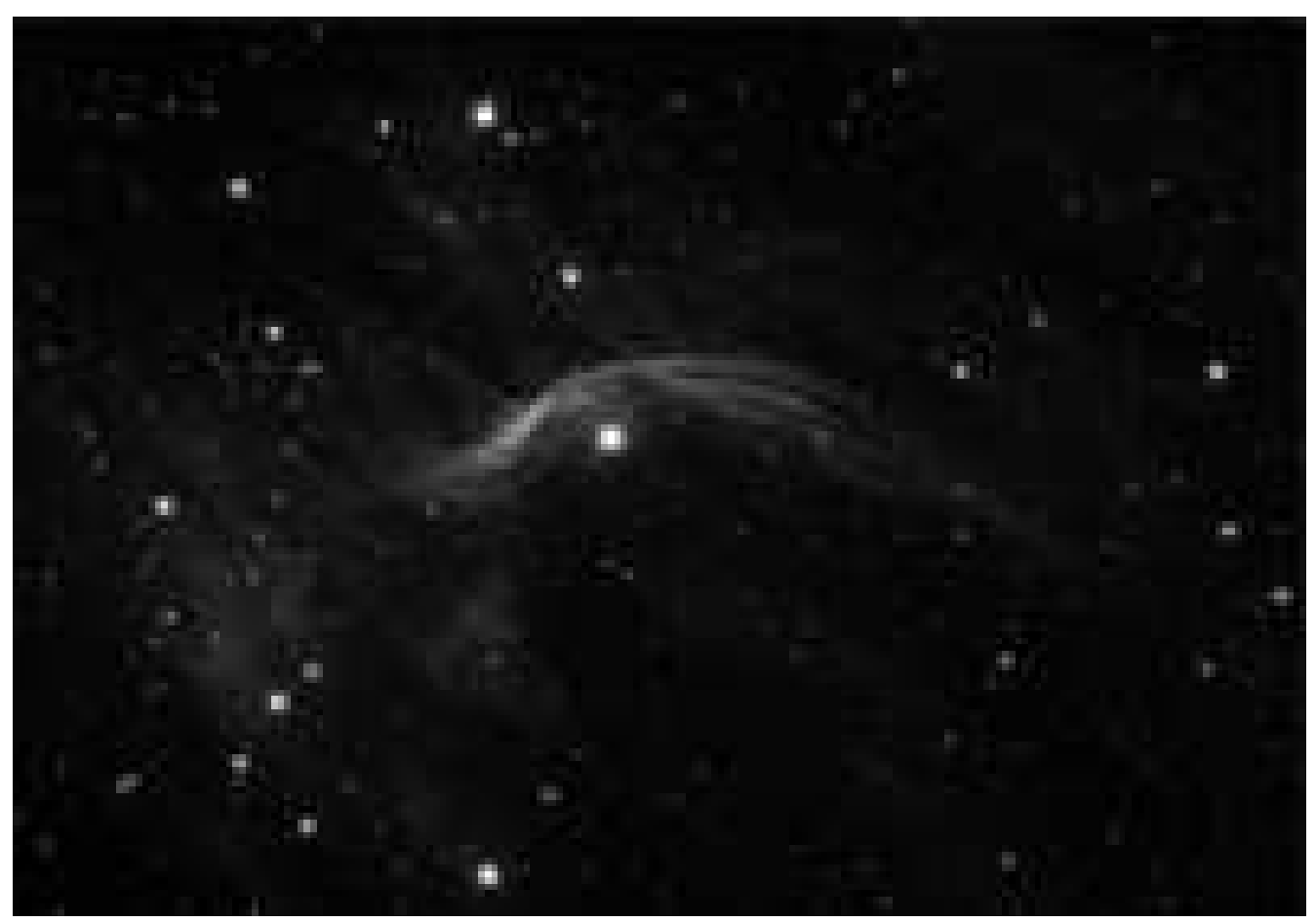

Figure 13. WISE $3.4 \mu \mathrm{m}$ (band W1, Wright et al. 2010) observation of the stellar wind bow shock surrounding the massive runaway O9.5 V star $\zeta$ Ophiuchi. The image represents about 35 arcmin in the horizontal direction, which at a distance of $112 \mathrm{pc}$ corresponds to about $1.12 \mathrm{pc}$.

with anisotropic thermal transfers on a field-aligned, magnetohydrodynamical bow shock flow around an OB-type, fast-moving star. We then explored the effects of the stellar motion with respect to the bow shocks, focusing on an initially $20 \mathrm{M}_{\odot}$ star moving with velocities $v_{\star}=20,40$ and $70 \mathrm{~km} \mathrm{~s}^{-1}$. We presented additional models of an initially $10 \mathrm{M}_{\odot}$ star moving with velocities $v_{\star}=40 \mathrm{~km} \mathrm{~s}^{-1}$ and of an initially $40 \mathrm{M}_{\odot}$ star moving with velocities $v_{\star}=70 \mathrm{~km} \mathrm{~s}^{-1}$. The ISM magnetic field strength is set to $B_{\text {ISM }}=7 \mu \mathrm{G}$. We also considered bow shock nebulae produced within a weaker ISM magnetic field $\left(B_{\mathrm{ISM}}=3.5 \mu \mathrm{G}\right)$. The other ISM properties are unchanged for each models.

Our models show that although the magnetization of the ISM does not radically change the global aspect of our bow shock nebulae, it slightly modifies their internal organiation. Anisotropic thermal transfers do not split the region of shocked ISM gas as in our hydrodynamical models (Paper I), since the presence of the magnetic field in the regions of shocked material forbids heat conduction perpendicular to the magnetic field lines. The field lines, initially parallel to the direction of stellar motion, are bent round by the bow shock into a sheath around the fast stellar wind bubble. As showed in Heitsch et al. (2007), the presence of the magnetic field stabilises the contact discontinuities inhibiting the growth of the Kelvin-Helmholtz instabilities that typically occur in pure hydrodynamical models at the interface between shocked ISM and shocked stellar wind.

As in our previous hydrodynamical study (Paper I), bow shocks are brighter in infrared reprocessed starlight. Their emission by optically-thin radiation mostly originates from the shocked ISM and their [OIII] $\lambda 5007$ spectral line emission are higher than their $\mathrm{H} \alpha$ emission. Notably, their X-rays emission are negligible compared to their optical luminosity and therefore it does not constitute the best waveband to search for hot massive stars' stellar wind bow shocks. We find that the presence of an ISM background mag- netic field has the effect of reducing the optical synthetic emission maps of our models, making them fainter by one and two orders of magnitude at [OIII] $\lambda 5007$ and $\mathrm{H} \alpha$, respectively. This may explain why not so many of them are observed at these spectral lines. We confirm that, under our assumptions and even in the presence of a magnetic field, circumstellar structures produced by high-mass, slowly-moving stars are the easiest observable bow shock nebulae in the warm neutral phase of the Milky Way.

We performed dust continuum radiative transfer calculations of our bow shocks models (cf. Acreman et al. 2016) and generated spectral energy distributions and isophotal emission maps for different wavelengths $25 \leqslant \lambda \leqslant 100 \mu \mathrm{m}$ and viewing angles $0^{\circ} \leqslant \phi \leqslant 90^{\circ}$. Consistently with the observation of van Buren \& McCrav (1988a); van Buren et al. (1995); Noriega-Crespo et al. (1997), the calculations show that our bow shocks are brighter at $60 \mu \mathrm{m}$. The projected infrared emission can also be diminished the presence of the ISM magnetic field, in particular at wavelengths $\lambda \geqslant 60 \mu \mathrm{m}$, since the amount of dust trapped into the bow shock is smaller. We also notice that the change in surface brightness of our emission maps as a function of the viewing angle of the bow shock is similar as in the optical waveband, i.e. it is brighter if $\phi=0^{\circ}$ and fainter if $\phi=90^{\circ}$ ( see Meyer et al. 2016).

In future models, we would like to extend this pioneering study of massive stars' bow shocks within the magnetized ISM towards three-dimensional models in which the ISM magnetic field is unaligned with respect to the motion of the star, as it has been done in order to appreciate its influence on the morphology of the global heliopause (Pogorelov \& Matsuda 1998). Such simulations will help to better understand the structure of the circumstellar nebulae forming around hot, ionising, massive runaway stars and allow us to predict more accurately, e.g. the optical emission signatures of these bow shocks. Thorough comparison with particular hot, bow- 
shock-producing massive stars, e.g. $\zeta$ Ophiuchi, might then be feasible.

\section{ACKNOWLEDGEMENTS}

The authors thank Tom Hartquist, for his kind help and very helpful advices when reviewing this paper and Richard Stancliffe for numerous grammatical comments when carefully reading the manuscript. D. M.-A. Meyer gratefully thanks T. Robitaille and C. Dullemond for their support with the HYPERION and the RADMC3D raditative transfer codes, respectively, as well as D. Thun for fruitful discussion concerning his master thesis. Are also acknowledged Prof. R. Jalabert and G. Weick from the Institute of Physics and Chemistry of Materials of Strasbourg (IPCMS) for their kind help concerning the Mie theory. This study was in parts conducted within the Emmy Noether research group on "Accretion Flows and Feedback in Realistic Models of Massive Star Formation" funded by the German Research Foundation under grant no. KU 2849/3-1. The authors gratefully acknowledge the computing time provided on the supercomputer JUROPA at Jülich Supercomputing Centre (JSC) and on the bwGrid cluster Tübingen. This research has made use of "Aladin sky atlas" and "VizieR catalogue access tool" both developed at CDS, Strasbourg Observatory, France.

\section{REFERENCES}

Acreman D. M., Stevens I. R., Harries T. J., 2016, MNRAS, 456, 136

Alexiades V., Amiez G., Gremaud P.-A., 1996, Communication in Numerical Methods in Engineering, 12, 31

Arnaud K. A., 1996, in Jacoby G. H., Barnes J., eds, Astronomical Data Analysis Software and Systems V Vol. 101 of Astronomical Society of the Pacific Conference Series, XSPEC: The First Ten Years. p. 17

Arthur S. J., Hoare M. G., 2006, ApJS, 165, 283

Asplund M., Grevesse N., Sauval A. J., Scott P., 2009, ARA\&A, 47, 481

Balsara D. S., Tilley D. A., Howk J. C., 2008, MNRAS, 386, 627

Baranov V. B., Barmin A. A., Pushkar' E. A., 1996, Astronomy Letters, 22, 555

Baranov V. B., Krasnobaev K. V., Onishchenko O. G., 1975, Soviet Astronomy Letters, 1, 81

Baranov V. B., Malama Y. G., 1993, J. Geophys. Res., 98, 15157

Bjorkman J. E., Wood K., 2001, ApJ, 554, 615

Blondin J. M., Koerwer J. F., 1998, New Ast., 3, 571

Bohren C. F., Huffman D. R., 1983, Absorption and scattering of light by small particles

Boley A. C., Morris M. A., Desch S. J., 2013, ApJ, 776, 101

Brighenti F., D'Ercole A., 1994, MNRAS, 270, 65

Brighenti F., D'Ercole A., 1995a, MNRAS, 277, 53

Brighenti F., D'Ercole A., 1995b, MNRAS, 273, 443

Brott I., de Mink S. E., Cantiello M., Langer N., de Koter A., Evans C. J., Hunter I., Trundle C., Vink J. S., 2011, A\&A, 530, A115

Brown D., Bomans D. J., 2005, A\&A, 439, 183

Burlaga L. F., Florinski V., Ness N. F., 2015, ApJ, 804, L31

Castor J. I., Abbott D. C., Klein R. I., 1975, ApJ, 195, 157

Chalov S. V., Malama Y. G., Alexashov D. B., Izmodenov V. V., 2016, MNRAS, 455, 431

Chiotellis A., Boumis P., Nanouris N., Meaburn J., Dimitriadis G., 2016, MNRAS, 457, 9

Chiotellis A., Schure K. M., Vink J., 2012, A\&A, 537, A139

Chita S. M., Langer N., van Marle A. J., García-Segura G., Heger A., 2008, A\&A, 488, L37

Churchwell E., Povich M. S., Allen D., Taylor M. G., Meade M. R., Babler B. L., Indebetouw R., Watson C., Whitney B. A., Wolfire M. G., Bania T. M., Benjamin R. A., Clemens 2006, ApJ, 649, 759

Comerón F., Kaper L., 1998, A\&A, 338, 273
Cowie L. L., McKee C. F., 1977, ApJ, 211, 135

Cox C. I., Gull S. F., Green D. A., 1991, MNRAS, 250, 750

Cox N. L. J., Kerschbaum F., van Marle A. J., Decin L., Ladjal D., Mayer A., 2012, A\&A, 543, C1

Crutcher R. M., Roberts D. A., Troland T. H., Goss W. M., 1999, ApJ, 515,275

de Jager C., Nieuwenhuijzen H., van der Hucht K. A., 1988, A\&AS, 72, 259

de Mink S. E., Cantiello M., Langer N., Pols O. R., Brott I., Yoon S.-C., 2009, A\&A, 497, 243

de Mink S. E., Pols O. R., Hilditch R. W., 2007, A\&A, 467, 1181

de Sterck H., Low B. C., Poedts S., 1998, Physics of Plasmas, 5, 4015

de Sterck H., Poedts S., 1999, A\&A, 343, 641

de Sterck H., Poedts S., 2000, in Verheest F., Goossens M., Hellberg M. A., Bharuthram R., eds, Waves in Dusty, Solar, and Space Plasmas Vol. 537 of American Institute of Physics Conference Series, Disintegration and reformation of intermediate shock segments in 3D MHD bow shock flows. pp 232-239

de Sterck H., Poedts S., 2001, in Freisthler H., Warnecke G. m. . f. p. .., eds, Waves in Dusty, Solar, and Space Plasmas Vol. 141 of International Series of Numerical Mathematics, Overcompressive shocks and compound shocks in 2D and 3D magnetohydrodynamic flows

del Valle M. V., Romero G. E., Santos-Lima R., 2015, MNRAS, 448, 207

Diaz-Miller R. I., Franco J., Shore S. N., 1998, ApJ, 501, 192

Donati J.-F., Babel J., Harries T. J., Howarth I. D., Petit P., Semel M., 2002, MNRAS, 333, 55

Dopita M. A., 1973, A\&A, 29, 387

Draine B. T., 2011, Physics of the Interstellar and Intergalactic Medium

Draine B. T., Lee H. M., 1984, ApJ, 285, 89

Dullemond C. P., , 2012, RADMC-3D: A multi-purpose radiative transfer tool, Astrophysics Source Code Library

Dyson J. E., 1975, Ap\&SS, 35, 299

Eldridge J. J., Genet F., Daigne F., Mochkovitch R., 2006, MNRAS, 367, 186

Eldridge J. J., Langer N., Tout C. A., 2011, MNRAS, 414, 3501

Esquivel A., Raga A. C., Cantó J., Rodríguez-González A., López-Cámara

D., Velázquez P. F., De Colle F., 2010, ApJ, 725, 1466

Evans K. A., Massey P., 2015, AJ, 150, 149

Fiedler R. A., Mouschovias T. C., 1993, ApJ, 415, 680

Fitzenreiter R. J., Scudder J. D., Klimas A. J., 1990, J. Geophys. Res., 95, 4155

Florinski V., Pogorelov N. V., Zank G. P., Wood B. E., Cox D. P., 2004, ApJ, 604, 700

Gaensler B. M., 1998, ApJ, 493, 781

Golub L., Pasachoff J. M., 1997, The Solar Corona, Cambridge University Press

Gull T. R., Sofia S., 1979, ApJ, 230, 782

Gvaramadze V. V., Langer N., Mackey J., 2012, MNRAS, 427, L50

Gvaramadze V. V., Menten K. M., Kniazev A. Y., Langer N., Mackey J., Kraus A., Meyer D. M.-A., Kamiński T., 2014, MNRAS, 437, 843

Harten A., Lax P. D., van Leer B., 1983, SIAM Review, 25, 35

Harvey-Smith L., Madsen G. J., Gaensler B. M., 2011, ApJ, 736, 83

Heger A., Woosley S. E., Spruit H. C., 2005, ApJ, 626, 350

Heiligman G. M., 1980, MNRAS, 191, 761

Heitsch F., Slyz A. D., Devriendt J. E. G., Hartmann L. W., Burkert A., 2007, ApJ, 665, 445

Huthoff F., Kaper L., 2002, A\&A, 383, 999

Kaper L., van Loon J. T., Augusteijn T., Goudfrooij P., Patat F., Waters L. B. F. M., Zijlstra A. A., 1997, ApJ, 475, L37

Kawamura A. D., Heerikhuisen J., Pogorelov N. V., 2010, AGU Fall Meeting Abstracts

Keppens R., Tóth G., Westermann R. H. J., Goedbloed J. P., 1999, Journal of Plasma Physics, 61, 1

Kobulnicky H. A., Chick W. T., Schurhammer D. P., Andrews J. E., Povich M. S., Munari S. A., Olivier G. M., Sorber R. L., Wernke H. N., Dale D. A., Dixon D. M., 2016, ArXiv e-prints:1609.02204

Kroupa P., 2001, MNRAS, 322, 231

Kudritzki R. P., Pauldrach A., Puls J., Abbott D. C., 1989, A\&A, 219, 205 
Kudritzki R.-P., Puls J., 2000, ARA\&A, 38, 613

Langer N., 2012, ARA\&A, 50, 107

Langer N., García-Segura G., Mac Low M.-M., 1999, ApJ, 520, L49

Linde T. J., Gombosi T. I., Roe P. L., Powell K. G., Dezeeuw D. L., 1998, J. Geophys. Res., 103, 1889

Lodders K., 2003, ApJ, 591, 1220

Lopez R. E., Merkin V. G., Lyon J. G., 2011, Annales Geophysicae, 29, 1129

Lucy L. B., 1999, A\&A, 344, 282

Mac Low M.-M., van Buren D., Wood D. O. S., Churchwell E., 1991, ApJ, 369,395

Mackey J., Haworth T. J., Gvaramadze V. V., Mohamed S., Langer N.,

Harries T. J., 2016, A\&A, 586, A114

Mackey J., Langer N., Gvaramadze V. V., 2013, MNRAS

Mackey J., Mohamed S., Gvaramadze V. V., Kotak R., Langer N., Meyer D. M.-A., Moriya T. J., Neilson H. R., 2014, Nature, 512, 282

Mackey J., Mohamed S., Neilson H. R., Langer N., Meyer D. M.-A., 2012, ApJ, 751, L10

Marchant P., Langer N., Podsiadlowski P., Tauris T. M., Moriya T. J., 2016, A\&A, 588, A50

Martins F., Genzel R., Hillier D. J., Eisenhauer F., Paumard T., Gillessen S., Ott T., Trippe S., 2007, A\&A, 468, 233

Mathis J. S., Rumpl W., Nordsieck K. H., 1977, ApJ, 217, 425

McComas D. J., Bzowski M., Frisch P., Fuselier S. A., Kubiak M. A., Kucharek H., Leonard T., Möbius E., Schwadron N. A., Sokół J. M., Swaczyna P., Witte M., 2015, ApJ, 801, 28

Meyer D. M.-A., Gvaramadze V. V., Langer N., Mackey J., Boumis P.,

Mohamed S., 2014, MNRAS, 439, L41

Meyer D. M.-A., Langer N., Mackey J., Velázquez P. F., Gusdorf A., 2015, MNRAS, 450, 3080

Meyer D. M.-A., Mackey J., Langer N., Gvaramadze V. V., Mignone A., Izzard R. G., Kaper L., 2014, MNRAS, 444, 2754

Meyer D. M.-A., van Marle A.-J., Kuiper R., Kley W., 2016, MNRAS, 459, 1146

Meyer D. M.-A., Vorobyov E. I., Kuiper R., Kley W., 2016, ArXiv eprints: 1609.03402

Mignone A., Bodo G., Massaglia S., Matsakos T., Tesileanu O., Zanni C., Ferrari A., 2007, ApJS, 170, 228

Mignone A., Zanni C., Tzeferacos P., van Straalen B., Colella P., Bodo G., 2012, ApJS, 198, 7

Mohamed S., Mackey J., Langer N., 2012, A\&A, 541, A1

Moore B. D., Walter D. K., Hester J. J., Scowen P. A., Dufour R. J., Buckalew B. A., 2002, AJ, 124, 3313

Neugebauer G., Habing H. J., van Duinen R., Aumann H. H., Baud B., Beichman C. A., Beintema D. A., Boggess N., Clegg P. E., de Jong T., Emerson J. P., Gautier T. N., Gillett F. C., Harris S., Hauser M. G., Houck J. R., Jennings R., 1984, ApJ, 278, L1

Noriega-Crespo A., van Buren D., Cao Y., Dgani R., 1997, AJ, 114, 837

Noriega-Crespo A., van Buren D., Dgani R., 1997, AJ, 113, 780

Ohno H., Shibata S., 1993, MNRAS, 262, 953

Opher M., Bibi F. A., Toth G., Richardson J. D., Izmodenov V. V., Gombosi T. I., 2009, Nature, 462, 1036

Orlando S., Bocchino F., Reale F., Peres G., Pagano P., 2008, ApJ, 678, 274

Orlando S., Peres G., Reale F., Bocchino F., Rosner R., Plewa T., Siegel A., 2005, A\&A, 444, 505

Osterbrock D. E., Bochkarev N. G., 1989, Soviet Ast., 33, 694

Parker E. N., 1958, ApJ, 128, 664

Parker E. N., 1963, Interplanetary dynamical processes. New York, Interscience Publishers

Pavlyuchenkov Y. N., Kirsanova M. S., Wiebe D. S., 2013, Astronomy Reports, 57, 573

Paxton B., Bildsten L., Dotter A., Herwig F., Lesaffre P., Timmes F., 2011, ApJS, 192, 3

Peri C. S., Benaglia P., Brookes D. P., Stevens I. R., Isequilla N. L., 2012, A\&A, 538, A108

Peri C. S., Benaglia P., Isequilla N. L., 2015, A\&A, 578, A45

Petrovic J., Langer N., Yoon S.-C., Heger A., 2005, A\&A, 435, 247
Pogorelov N. V., Matsuda T., 1998, J. Geophys. Res., 103, 237

Pogorelov N. V., Matsuda T., 2000, A\&A, 354, 697

Pogorelov N. V., Semenov A. Y., 1997, A\&A, 321, 330

Povich M. S., Benjamin R. A., Whitney B. A., Babler B. L., Indebetouw

R., Meade M. R., Churchwell E., 2008, ApJ, 689, 242

Raga A. C., 1986, ApJ, 300, 745

Raga A. C., Cantó J., De Colle F., Esquivel A., Kajdic P., RodríguezGonzález A., Velázquez P. F., 2008, ApJ, 680, L45

Raga A. C., Noriega-Crespo A., Cantó J., Steffen W., van Buren D., Mellema G., Lundqvist P., 1997, Rev. Mex. Ast., 33, 73

Rand R. J., Kulkarni S. R., 1989, ApJ, 343, 760

Ratkiewicz R., Barnes A., Molvik G. A., Spreiter J. R., Stahara S. S., Vinokur M., Venkateswaran S., 1998, A\&A, 335, 363

Richardson I. G., 2016, ArXiv e-prints:1603.06137

Robitaille T. P., 2011, A\&A, 536, A79

Rozyczka M., Tenorio-Tagle G., 1995, MNRAS, 274, 1157

Sana H., de Mink S. E., de Koter A., Langer N., Evans C. J., Gieles M., Gosset E., Izzard R. G., Le Bouquin J.-B., Schneider F. R. N., 2012, Science, 337, 444

Sexton R. O., Povich M. S., Smith N., Babler B. L., Meade M. R., Rudolph A. L., 2015, MNRAS, 446, 1047

Shabala S. S., Mead J. M. G., Alexander P., 2010, MNRAS, 405, 1960

Sharpless S., 1959, ApJS, 4, 257

Simpson R. J., Povich M. S., Kendrew S., Lintott C. J., Bressert E., Arvidsson K., Cyganowski C., Maddison S., Schawinski K., Sherman R., Smith A. M., Wolf-Chase G., 2012, MNRAS, 424, 2442

Soker N., Dgani R., 1997, ApJ, 484, 277

Spitzer L., 1962, Physics of Fully Ionized Gases

Thun D., Kuiper R., Schmidt F., Kley W., 2016, A\&A, 589, A10

Troland T. H., Heiles C., 1986, ApJ, 301, 339

van Buren D., McCray R., 1988a, ApJ, 329, L93

van Buren D., McCray R., 1988b, ApJ, 329, L93

van Buren D., Noriega-Crespo A., Dgani R., 1995, AJ, 110, 2914

van Marle A. J., 2006, PhD thesis, Utrecht University

van Marle A. J., Decin L., Meliani Z., 2014, A\&A, 561, A152

van Marle A. J., Langer N., Achterberg A., García-Segura G., 2006, A\&A, 460,105

van Marle A. J., Langer N., García-Segura G., 2007, A\&A, 469, 941

van Marle A. J., Meliani Z., Keppens R., Decin L., 2011, ApJ, 734, L26

van Marle A. J., Meliani Z., Marcowith A., 2015, A\&A, 584, A49

Velázquez P. F., Martinell J. J., Raga A. C., Giacani E. B., 2004, ApJ, 601, 885

Viallet M., Baty H., 2007, A\&A, 473, 1

Villaver E., Manchado A., García-Segura G., 2012, ApJ, 748, 94

Vink J. S., 2006, in Lamers H. J. G. L. M., Langer N., Nugis T., Annuk K., eds, Stellar Evolution at Low Metallicity: Mass Loss, Explosions, Cosmology Vol. 353 of Astronomical Society of the Pacific Conference Series, Massive star feedback - from the first stars to the present. p. 113 Wachter S., Mauerhan J. C., Van Dyk S. D., Hoard D. W., Kafka S., Morris P. W., 2010, AJ, 139, 2330

Wang C., Han J. P., Li H., Peng Z., Richardson J. D., 2014, Journal of Geophysical Research (Space Physics), 119, 6199

Wareing C. J., Zijlstra A. A., O'Brien T. J., 2007a, MNRAS, 382, 1233

Wareing C. J., Zijlstra A. A., O’Brien T. J., 2007b, ApJ, 660, L129

Washimi H., Tanaka T., 1996, Space Sci. Rev., 78, 85

Weaver R., McCray R., Castor J., Shapiro P., Moore R., 1977, ApJ, 218, 377

Webber W. R., 2016, ArXiv e-prints:1604.06477

Wilkin F. P., 1996, ApJ, 459, L31

Wolfire M. G., McKee C. F., Hollenbach D., Tielens A. G. G. M., 2003, ApJ, 587, 278

Wood K., Whitney B. A., Robitaille T., Draine B. T., 2008, ApJ, 688, 1118 Wright E. L., Eisenhardt P. R. M., Mainzer A. K., Ressler M. E., Cutri R. M., Jarrett T., Kirkpatrick J. D., Padgett D., McMillan R. S., Skrutskie M., Stanford S., 2010, AJ, 140, 1868

Yoon S.-C., Cantiello M., 2010, ApJ, 717, L62

Yoon S.-C., Langer N., 2005, A\&A, 443, 643

Zank G. P., 2015, ARA\&A, 53, 449 
Zank G. P., Pogorelov N. V., Heerikhuisen J., Washimi H., Florinski V., Borovikov S., Kryukov I., Müller H. R., 2009, Space Sci. Rev., 146, 295 Zhu F.-Y., Zhu Q.-F., Li J., Zhang J.-S., Wang J.-Z., 2015, ApJ, 812, 87 